\title{
TRAIANO E LA COSTRUZIONE DELLA SUA IMMAGINE NEL FORO
}

\section{TRAJAN AND THE CONSTRUCTION OF HIS REPRESENTATION IN THE FORUM}

\author{
LUCREZIA UNGARO \\ Sovrintendenza Capitolina ai Beni Culturali del Comune di Roma \\ Museo dei Fori Imperiali \\ lucrezia.ungaro@comune.roma.it \\ ORCID: 0000-0002-2209-7953
}

DOI: $10.1387 /$ veleia.19668

Riassunto: La scoperta di un nuovo ritratto colossale di Traiano avvenuta durante la preparazione della mostra "Traiano. Costruire l'Impero, creare l'Europa», produce una rinnovata lettura del complesso programma figurativo voluto dall'imperatore nel suo Foro. Nel quadro della sua azione politica, militare e sociale, il Foro è infatti la massima rappresentazione della sua virtus imperatoria e della maiestas populi romani. In particolare, vengono riconsiderati i ritratti del Traianus Pater e della cosiddetta Agrippina/Marcia, alla luce di una possibile galleria dedicata alla famiglia genetica di Traiano e ai suoi modelli, come Giulio Cesare. Pari attenzione si dedica alla distribuzione delle sculture e dei rilievi noti negli spazi forensi, al loro rapporto gerarchico nello spazio sovradimensionato della piazza. Da ultimo, viene ripresa la proposta di riconoscere nell'aula trisegmentata la porticus porphiretica, riesaminando in via preliminare le sculture note in porfido attribuibili al Foro, e alcuni frammenti conservati nei depositi del Museo dei Fori Imperiali che acquisiscono così nuovo interesse.

Parole chiave: nuovo ritratto colossale di Traiano, Agrippina/Marcia, Traianus Pater, programma figurativo del Foro, virtus imperatoria, la famiglia imperiale, porticus porphiretica.

Abstract: The discovery of a new colossal portrait of Trajan occurred during the preparation of the exhibition "Trajan. Building the Empire, creating Europe», gave a renewed reading of the complex figurative program wanted by the emperor in his Forum. In the framework of his political, military and social action, the Forum is in fact the highest representation of his virtus imperatoria and of the maiestas populi romani. In particular, the portraits of the Traianus Father and of the so-called Agrippina / Marcia are reconsidered, in the light of a possible gallery dedicated to Trajan's genetic family and his models, such as Julius Caesar. Equal attention is devoted to the distribution of sculptures and reliefs discovered in forensic spaces, to their hierarchical relationship in the huge space of the square. Finally, the proposal to recognize the porticus porphiretica in the three-segmented hall is reconsidered, examining preliminarily the known porphyry sculptures attributable to the Forum, and some fragments preserved in the deposits of the Museum of the Imperial Fora, thus getting new interest.

Keywords: New colossal portrait of Trajan, Agrippina/Marcia, Traianus Pater, new figurative plan of the Forum, virtus imperatoria, Imperial family, porticus porphiretica. 
La diffusa ispirazione al ritratto di Traiano in varie e lontane località dell'Impero, testimonia quanto l'imperatore sia stato attento alla diffusione della sua immagine ${ }^{1}$, utilizzando tutti i media dell'epoca: le legioni stesse, portatrici di insegne e stendardi, di tabulae pictae, di ritratti veri e propri, insieme agli altri due mezzi di comunicazione per eccellenza, le monete ${ }^{2}$ e le sculture collocate nei luoghi pubblici di maggior importanza e frequentazione per la vita cittadina ${ }^{3}$.

Non è l'obiettivo di questo intervento ripercorrere la letteratura sulle origini della famiglia di Traiano, ma alla luce delle interpretazioni e recenti scoperte sull'apparato figurativo del Foro, questa può acquistare un ruolo sempre più determinante nel suo programma figurativo.

E' significativo che vi siano revisioni critiche su questo argomento molto diverse. F. Chausson sottolinea che non vi è certezza sul luogo di nascita di Traiano; il padre Marcus Ulpius Traianus è però di certo un nativo di Italica in Betica, ma le iscrizioni locali danno più informazioni sui Trahii piuttosto che sugli Ulpii. Il cognomen Traianus potrebbe derivare dal gentilizio Trahius ${ }^{4}$. L'altra questione problematica è nelle origini della madre, della quale non si hanno riferimenti certi nelle fonti, ma sulla base del nome della sorella di Traiano, Marciana, che potrebbe derivare dai Marcii, si desume, in modo un po' troppo semplicistico secondo F. Chausson, che la madre si sia chiamata Marcia; inoltre, non si può in realtà affermare con certezza che la madre (o la nonna) di Traiano sia stata di origine iberica. Anche per quanto riguarda le origini della moglie dell'imperatore, Plotina, non vi sono certezze: è probabile un suo imparentamento sia con Traiano da un lato, sia con il suo successore, Adriano, dall'altro, secondo la logica del rafforzamento dei legami familiari (quindi, delle proprietà e delle ricchezze) ${ }^{5}$. Per F. Chausson l'adozione è una finzione storiografica, come la «scelta del migliore» è un mito storiografico, evidentemente esaltato dal Panegirico di Plinio il Giovane declamato in senato il primo settembre del 100 d.C.: Nerva subisce le forti pressioni politiche del senato, Traiano è un militare forte dell'appoggio incondizionato delle sue truppe ed ha alle spalle un solido clan familiare tra la Betica, la Narbonense e l'Italia ${ }^{6}$ e a Roma i tempi sono maturi per accettare un imperatore externus, che, insieme alla moglie Plotina, inaugura un modus vivendi diverso rispetto al passato, giungendo nella capitale quasi due anni dopo la proclamazione, a piedi in testa alle sue truppe ${ }^{7}$.

Il programma del nuovo imperatore è poliedrico: philopolémos («amante della guerra») ${ }^{8}$, providentissimus ${ }^{9}$ e optimus princeps al tempo stesso, amministratore dello Stato, innovatore nel campo del welfare, dove hanno un ruolo rilevante le donne della sua famiglia, finanzia infrastrutture

1 Ringrazio di cuore Julián González per questa occasione: il presente contributo è un'estensione dell'articolo apparso nel catalogo della mostra «Traiano. Costruire l'Impero, creare l'Europa" (Roma, Mercati di Traiano, 29 novembre 2017-18 novembre 2018): Ungaro 2017 a, 91-97.

2 Cappellacci \& Molinari 2017, 88-90 per una efficace sintesi anche bibliografica.

3 Ruck 2007, 73-111; Cadario 2007, 95-102.

4 Chausson 2007, 125-133.

5 Piuttosto avventurosa la ricostruzione dell'albero genealogico proposta da Canto 2003, 46-57; l'A. è molto critica verso altri studiosi contemporanei che sembrano rifiutare in modo preconcetto l'origine ispanica di Traiano addirittura negando la sua presenza ad Italica: dimenticano che lui è stato comandante della Legio III di stanza in quella città per tre anni. Sulla po- litica dinastica di Traiano: Gonzáles-Conde Puente 2015, 127-148.

6 Chausson 2007, 140-143: non manca di sottolineare che a Selinus si ritrovano al capezzale di Traiano, Plotina, Adriano e il suo tutore Attiano.

7 Dione Cassio 68.23: «...aveva sempre marciato a piedi insieme a tutto l'esercito a testimonianza della condivisione della durezza della vita militare con i suoi commilitoni...».

8 Dione Cassio, 68.7.4. Altrettanto significativa la definizione moderna al superlativo bellicosissimus che si deve a Speidel 2002; cfr. Rinaldi Tufi 2017, 30-38.

9 Dall'iscrizione monumentale sull'arco di Ancona, nuovamente dedicato modificato nel 114 d.C., quando Traiano assume anche il titolo di Optimus Princeps. CIL IX, 5894. 
e opere pubbliche ovunque, e quando sembra agire spinto soprattutto da obiettivi militari, in realtà persegue anche l'espansione commerciale e il controllo delle rotte importanti verso oriente, nell'interesse della sicurezza e del conseguente benessere dell'Impero ${ }^{10}$.

Basta ricordare in sintesi la successione dei principali obiettivi raggiunti in una manciata di anni: dopo aver consolidato il confine renano, affronta le campagne daciche (101-102, 105106 d.C.); quella che porterà alla formazione della provincia Ethiopia (106 d.C.), determinante per il controllo delle rotte verso il Golfo Persico e per il controllo stabile della Valle del Nilo; otterrà senza spargimento di sangue la riorganizzazione dell'area medio orientale con la nuova provincia di Arabia Petraea (106 d.C.), strategica per lo sbocco sul Mar Rosso e la base logistica per le campagne partiche; il rafforzamento del limes africano per garantire il flusso di merci dal bacino del Niger ${ }^{11}$; seguirà la campagna partica tra il 113 d.C. e il 117 d.C., dal trionfo e dalla formazione delle nuove province al ritiro, tra le cui concause ricordiamo la rivolta giudaica («la guerra di Kitos») tra il 115 e il 117 d.C. ${ }^{12}$.

Le opere pubbliche sono il volano della sua azione divenendo «manifesto» del suo governo e della sua presenza: i Romani, grandi costruttori, applicano innovazioni tecnologiche che condizioneranno l'evoluzione di sistemi costruttivi ${ }^{13}$. I fasti consolari, che registrano la sua presenza alle inaugurazioni nell'urbe, e le emissioni monetali, che arrivano ovunque a testimoniare quanto viene realizzato nella e per la capitale, rappresentano il suo complesso «sistema» di governo, insieme all'incremento costante degli interventi nel welfare con un effetto positivo moltiplicatore del consenso ${ }^{14}$. Basta ricordare un dato: le ricchezze derivate dalla conquista della Dacia vengono investite anche nell' institutio alimentaria che passa da stanziamenti di 72.000 a 1 milione e 44.000 sesterzi ${ }^{15}$.

Traiano, che non ha discendenza dinastica e tantomeno divina, deve consolidare la sua figura e la sua immagine: i modelli di riferimento sono Alessandro Magno, Giulio Cesare ed Augusto, ma il perno è la sua famiglia a cominciare dai due padri, che verranno ambedue divinizzati ${ }^{16}$, e dalle donne che vivranno a corte, la moglie Plotina, la sorella Marciana, la nipote Matidia e le sue figlie, Matidia Minore e Sabina.

Il ruolo delle donne nel in secolo d.C. cambia attraverso la loro acquisizione dello ius imaginum e l'allargamento della domus imperiale con il conferimento del titolo di Augusta, in parallelo a quello di pater patriae: una visione della coppia imperiale e della loro discendenza impegnata nel sociale per il bene della collettività ${ }^{17}$.

Dilaga la sua autorappresentazione nelle tre cariche supreme, come capo militare che indossa la lorica dalle decorazioni simboliche, come pontefice massimo che presiede le più importanti cerimonie sacre, e come magistrato che garantisce la giustizia per tutti gli abitanti dell'impero. La sta-

10 Equini Schneider 2017, 50-56 per il corretto punto di vista soprattutto sulla spedizione partica.

11 Vismara 2017, 57-64.

12 Gonzales 2017, 65-68.

13 Tra tutti citiamo i risultati scientifici ottenuti nel corso dei restauri dei Mercati di Traiano: Ungaro 2010a, 5-28; Perucchio \& Brune 2010, 115-130; Speranza 2010, 131-144; Jackson et al. 2010, 145-153: dagli archi di contrasto realizzati nella Grande Aula dei Mercati di Traiano, inizio di un'importante evoluzione per i futuri grandi edifici termali, ai presidi antisismici, all'uso di una matrice cementizia che la rende «armata».
${ }_{14}$ Per la produzione monetale: Woytek 2010.

15 Rinaldi Tufi 2017, 31.

16 Per i precisi riferimenti monetali vedi oltre la nota 65. Significativo per la diffusione dell'immagine il ritratto di Traianus Pater in bronzo, rinvenuto presso un pilone del ponte sul Danubio tra Kostel (Pontes) e Turnu Severin (Drobeta), conservato presso il Narodni Muzeuj di Belgrado (inv. 2873 III; Ratković 2018, 135).

17 Wood 1999; Cenerini 2017, 107-112, sull'evoluzione del ruolo delle clarissimae foeminae a partire da Traiano; Balielo 2017, 100-106 sull'impegno delle donne della corte nel sociale. 
tua loricata riprende il modello augusteo del Mars Ultor nel quale la decorazione dei grifoni affrontati con thymiaterion nascente da palmetta si ricollega ai temi universali della propaganda imperiale e al carattere sacro apollineo, piuttosto che a quello vendicativo del dio ${ }^{18}$. Ma quest'ultimo aspetto si riaffaccia probabilmente nel I sec. d.C. con Traiano, che vendica le perdite di Domiziano, recupera insegne e armi, dedica il Tropeum Traiani di Adamclissi al dio Marte Ultore ${ }^{19}$. Dal Foro di Traiano provengono, come vedremo, due statue, una di togato in posa di magistrato, e una di loricato con i grifoni rappresentati sulla corazza, ma parzialmente coperti da un alto cingulum, secondo un modello microasiatico più antico ${ }^{20}$. Ambedue sono caratterizzate dal retro non finito che le fa immaginare collocate dentro nicchie o comunque contro muro ${ }^{21}$. Grande attenzione viene attribuita alla creazione dell'immagine ufficiale, il suo ritratto ha un'evoluzione molto controllata, inizia con tipi fisiognomici per evolversi verso quelli più idealizzati: fondamentale il ritratto ufficiale creato in occasione del trionfo dacico o per i dieci anni dall'acclamazione e comunque noto come il tipo IV o dei decennalia ${ }^{22}$ (fig. 1).

L'occasione per materializzare un vero programma di comunicazione è la sua massima realizzazione urbana, il Foro di Traiano con la Colonna coclide, dove la sua rappresentazione di primus inter pares, costantemente sottolineata, lo esalta quale massimo condottiero e stratega dell'esercito romano (che include tante forze armate provenienti da province e da popolazioni diverse). Vero trofeo urbano, il fusto istoriato rappresenta il monumento onorario dedicato ai suoi uomini e quello celebrativo per la sua apoteosi: la Colonna diviene così strumento per avvicinarsi alla sfera divina ${ }^{23}$.

In altre occasioni abbiamo osservato come progetto architettonico e programma figurativo debbano essere visti in un'unica visione organica e, se è già così nel Foro di Augusto, ancor più lo diventa nel Foro traianeo ${ }^{24}$ : le dimensioni del complesso "oversize», dilatate, e soprattutto della piazza, ne fanno uno spazio nuovo per l'esaltazione delle virtutes dell'imperatore e della maiestas imperii romani ${ }^{25}$. La virtus imperatoria è del resto il motivo centrale dell'attività edilizia, a maggior ragione nel Foro, dove il senato e il popolo romano dedicano statue digna memoratu in quanto Traiano è optime de re publica / merito domi forisque, come ricordano le iscrizioni qui rinvenute ${ }^{26}$, incise su basamenti per statue che in proporzione potrebbero essere alte tra 2,70/3,00 metri ${ }^{27}$ e che 368.

18 Cadario 2004, 109-120, 139-153, 247-260, 365-

19 Ungaro (scheda cat.), in Parisi Presicce et al. 2017, 391-392, n. 6 con bibliografia. Sul trofeo il messaggio è drastico, la sconfitta è senza appello, il monito chiaro: chi si oppone ai Romani verrà annientato. Traiano recupera le insegne perse da Domiziano e consuma la vendetta per riscattare i caduti: la scena XXV della Colonna Traiana prelude a tutto questo, cfr. Ungaro (scheda cat.), in Parisi Presicce et al. 2017, 403, n. 17 a, con bibliografia.

20 Cadario 2011, 105-113 e scheda cat. I, 1, 260: commento alla statua loricata da Copenaghen.

21 Ungaro (schede cat.), in Parisi Presicce et al. 2017, 391-393, nn. 6 e 7.

22 Si vedano a tal proposito per il tipo dei decennalia Colugnati (scheda cat.), in La Rocca $\&$ Parisi Presicce, 2011, 261, cat. I, 3; per quello con egida e corona civica Caporossi (scheda cat.), in La Rocca \& Parisi Presicce, 2011, 262, n. I, 4: un unicum di grande interesse in ambiente orientale. Da ultimo, Arata 2017 (scheda cat), in Parisi Presicce et al., cat.1, 387.
23 Coarelli 1999; Veyne 2007, 328-353; Ungaro 2014a, 67-85; Ungaro 2014b, 1485-1488.

24 Ungaro 2008, 399-417; Ungaro 2011, 43-62.

25 Velleio Patercolo già pone in relazione l'opera publica e la maiestas imperii Romani (Vell. 2,39,2); Scheithauer 2000, 245. Ruck 2007: l'importante monografia, oltre a codificare il concetto stesso di «colossalità», dando un quadro complessivo delle opere riferibili a tale "misura», le mette in relazione con lo spazio e l'architettura, ne esplicita il significato (22$119)$, le qualifica quale mezzo di rappresentazione (201).

26 Scheithauer 2000, 154-166: l'Autore mette in relazione le fonti letterarie con iscrizioni e basi iscritte relative al programma figurativo dei grandi complessi monumentali romani, con particolare attenzione al Foro traianeo. Le basi sono quelle conservate all'esterno dell'area della Basilica Ulpia (nn. inv. FT 2640, 2722, 3442; CIL VI, 959 b, 959 a, 959 c).

27 Non lontane dalle statue rinvenute nello scavo novecentesco e di cui si tratterà poco più avanti. 
dovevano trovarsi davanti alla facciata della Basilica Ulpia. Queste iscrizioni dedicatorie sono datate al 112 d.C. e ci dicono che il Senato aveva "deliberato» la dedica delle statue all'imperatore, quali parti integranti dell'apparato forense.

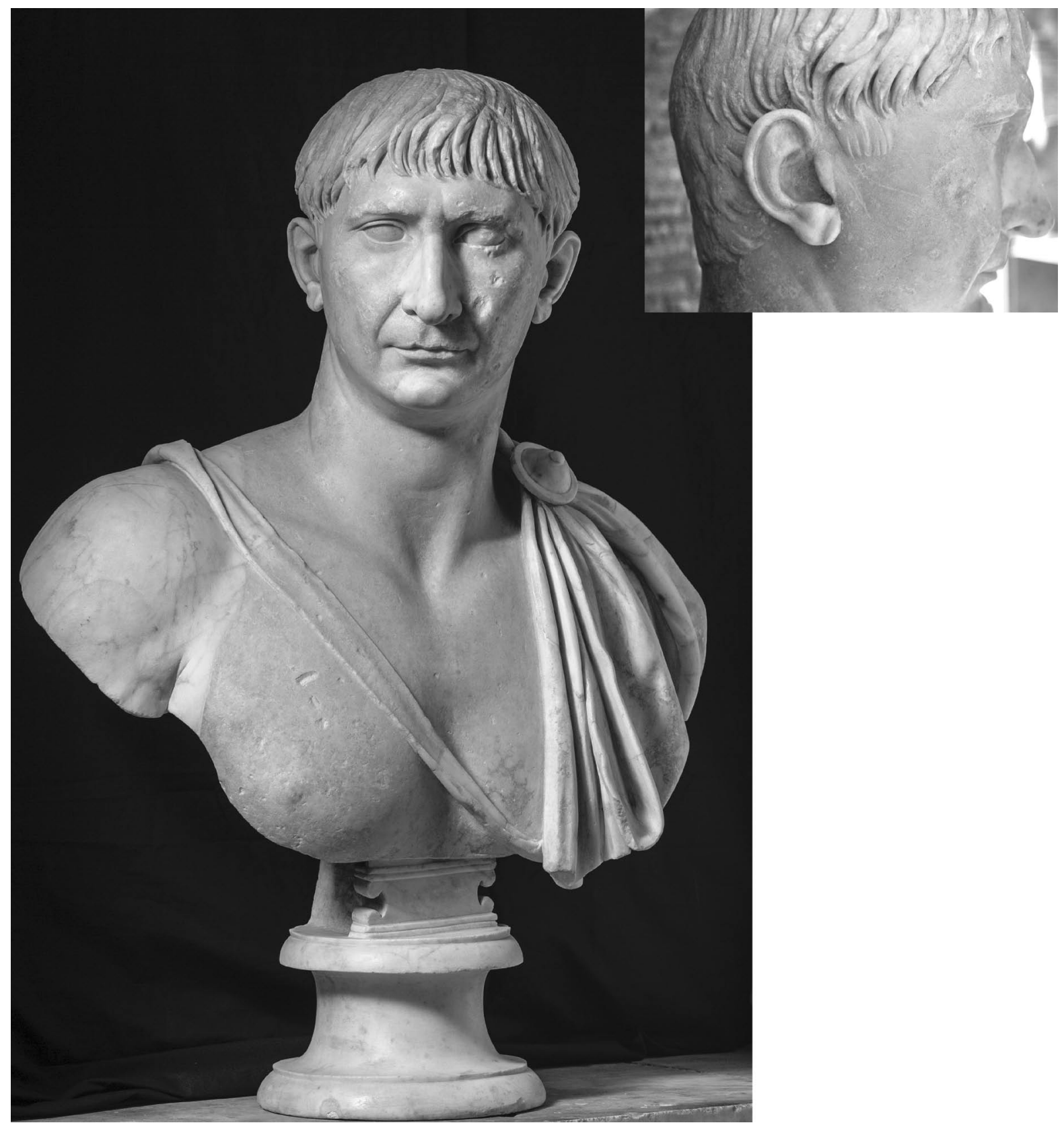

Fig. I. Ritratto di Traiano tipo decennalia. Musei Capitolini inv. MC 276. A: visione frontale. B: dettaglio del profilo lato destro. 


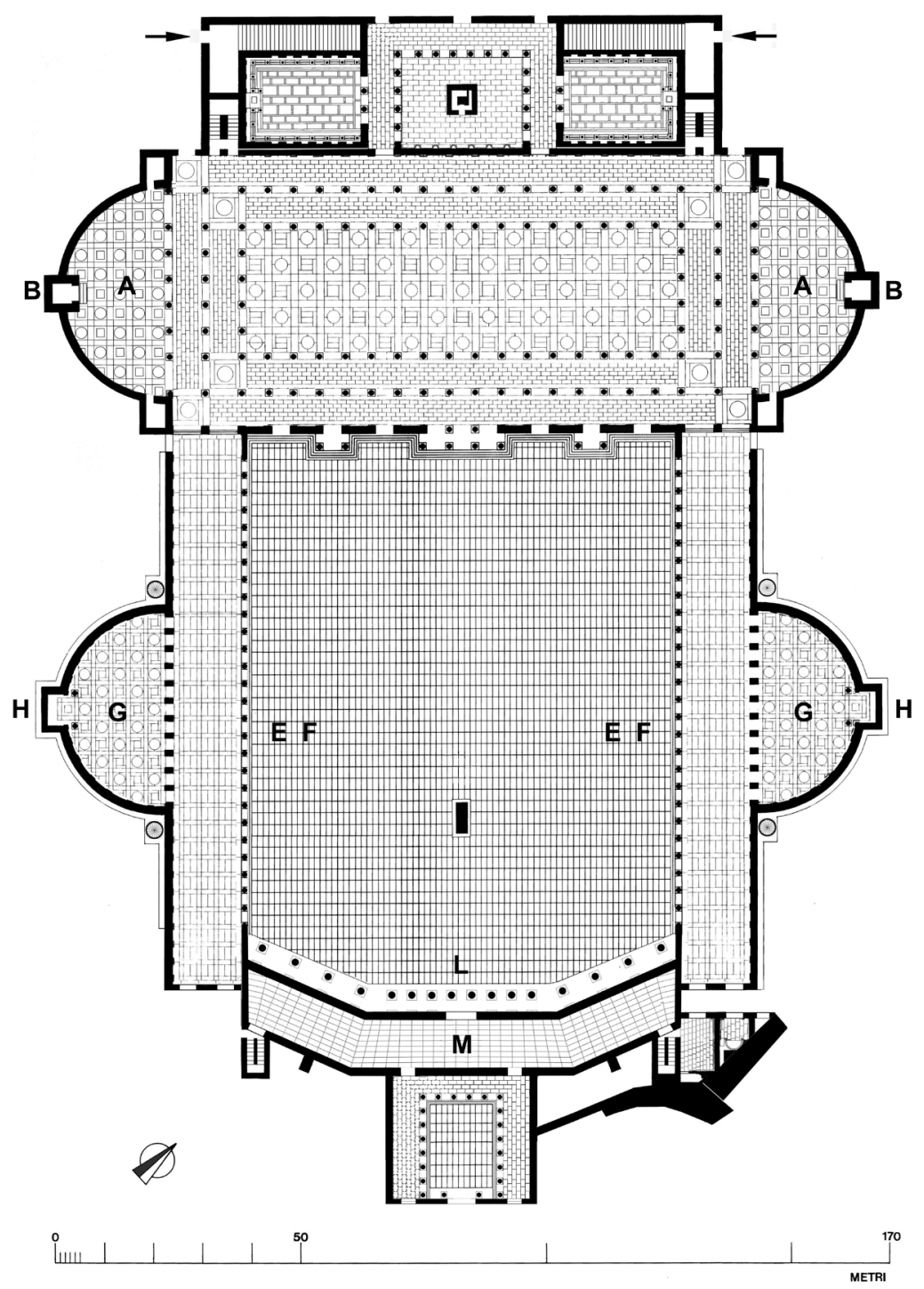

FIg. 2. Pianta del Foro di Traiano (Archivio Grafico Ufficio Fori Imperiali). Collocazione possibile di sculture negli edifici forensi in base agli attuali rinvenimenti: A. nicchie delle esedre della Basilica Ulpia, gallerie di statue colossali femminili della famiglia imperiale e ideali (h. $m$ 2,60 circa) B. nicchie al centro delle esedre della Basilica Ulpia, statue colossali imperiali (h. $m$ 4,80 e oltre?), statue di culto? C. facciata della Basilica Ulpia, attico, alternanza statue di Daci e altre popolazioni vinte in marmo bianco h. $m$ 2,60 circa, con pannelli raffiguranti cataste di armi, insegne delle legioni D. facciata della Basilica Ulpia, in corrispondenza degli ingressi basamenti recanti iscrizioni dedicatorie per le statue colossali dell'imperatore (h. presunta $m$ 2,70/3,00 circa) E. facciata portici sulla piazza del Foro, attico: alternanza statue colossali di Daci in marmo bianco e pavonazzetto h. $m 3$ circa, e specchiature contenenti imagines clipeatae con ritratti colossali $\mathbf{F}$. statue acroteriali di Daci elo ulteriori insegne $\mathbf{G}$. nicchie delle esedre dei portici, statue colossali di personaggi illustri h. m 2,80 circa H. nicchie al centro delle esedre, gruppi scultorei, statue colossali? L. facciata edificio meridionale, imagines clipeatae e statue nelle nicchie oppure ingresso al Foro lato nord, secondo l'attribuzione della rappresentazione sull'aureo coniato per il FORUM TRAIANI M. aula trisegmentata: pavimentazione in porfido, statue di Daci e vinti, statue imperiali, iscrizioni. 
La distribuzione delle sculture secondo significati e dimensioni diverse va quindi ricercata in sintonia alle funzioni dei luoghi, ai percorsi previsti, agli ordini architettonici e soprattutto tenendo conto dell'equilibrio tra vincitori, con il grande spiegamento di truppe, e vinti, presenti attraverso le molteplici statue dei Daci e associati alle congeries armorum che con Traiano invadono lo spazio pubblico e sono monumentalizzate ${ }^{28}$. La Colonna, la facciata della Basilica Ulpia e le facciate dei portici della piazza esaltano il ruolo delle legioni romane ${ }^{29}$, che hanno portato alla vittoria sui Daci e sulle altre popolazioni, e più in generale il potere dell'impero romano dai confini ormai pacificati lungo il limes renano-danubiano, e in altre aree strategiche come abbiamo accennato sopra. Per Traiano è quindi il luogo ideale dove consolidare l'immagine della famiglia e dei suoi modelli di riferimento, nonché circondarsi degli uomini illustri che hanno contribuito alla sua ascesa.

La parola ai reperti archeologici.

La storia degli scavi mostra la spoliazione dovuta prima allo sfruttamento del grande complesso come vera e propria cava urbana di marmi, poi a scavi più o meno fortuiti, in alcuni casi con dispersione del materiale, in altri con la conservazione in collezioni, fino agli sterri degli anni Trenta del secolo scorso dai quali emergono un certo numero di grandi frammenti e migliaia di piccoli (ma a volte preziosi, come vedremo) frammenti di vario genere ${ }^{30}$.

Il numero di statue e ritratti di sicura provenienza dal Foro è davvero esiguo e soprattutto ancora non è certo il programma completo di questo apparato scultoreo in stretta relazione con la tessitura architettonica degli edifici.

Gli spazi nei quali possiamo aspettarci che Traiano abbia voluto inserire cicli statuari sono abbastanza circoscrivibili: la Basilica Ulpia (le esedre con le nicchie centrali), le esedre dei portici, l'aula trisegmentata, forse anche la corte aperta verso il Foro di Augusto. Discorso a parte meritano le imagines clipeatae attestate come noto dalla moneta, che si pensa possa rappresentare la facciata oggi attribuita all'aula trisegmentata a sud, ma che non si può escludere possa raffigurare una sistemazione monumentale a nord ${ }^{31}$, e come vedremo sull'attico della facciata dei portici in base ad alcuni ritrovamenti (fig. 2).

Affrontiamo ora le opere venute alla luce nel secolo scorso, statue e ritratti, alcune opere attribuite al Foro ma oggi in diverse collezioni, e la loro possibile relazione con la rappresentazione dei vinti e delle congeries armorum.

Le immagini del XIX secolo ci restituiscono l'area scavata della Basilica Ulpia sotto il governo del papa Pio VII, quando nel cosiddetto «Museo delle rovine» vengono disposte a terra e lungo il muro perimetrale statue frammentarie di «Daci» nei due marmi principali, bianco e pavonazzetto, insieme a statue frammentarie forse appartenenti a gallerie di uomini illustri ${ }^{32}$. Nel xIX secolo viene scavato un breve tratto della strada basolata ai piedi dei Mercati di Traiano a sud ${ }^{33}$, ma non risultano emersi materiali di rilievo, mentre a partire dal 1926 iniziano i grandi sterri ad opera del Governatorato di Roma sotto la guida di Corrado Ricci: viene «liberato» tutto l'emiciclo orientale del

28 Ungaro 2017b, 146-150 sui popoli rappresentati. Ungaro 1995, 99-193; Ungaro 2002, 129-133; 334-339, schede cat. 32-35; Ungaro 2010b, 105113.

${ }_{29}$ Pastor 2017, 286-290. Il collega sta ampliando la ricerca all'apparato epigrafico e ai simboli delle varie formazioni militari sull'intera piazza forense.

30 Ungaro 1993, 129-174; Ungaro 2005, 69-86; Meneghini 2017, 257-262.
31 Boccardi (scheda cat.), in Parisi Presicce et al. 2017, 420, n. 27g e veduta acquerellata a cura di Roberto Meneghini (Meneghini 2009). In questo stesso volume Eugenio La Rocca.

32 Virlouvet 1985, 184-207, XVII, 187, fig. 11. Ungaro 1993, 129-131, fig. 27.

33 Scavo fondamentale per raggiungere la consapevolezza della distinzione e dell'autonomia tra il complesso in laterizi a ridosso delle pendici del Quirinale e il Foro vero e proprio. 
Foro. I Giornali dei Lavori redatti da Amleto Paroli ci permettono di riconoscere alcuni importanti reperti all'atto della scoperta. Si tratta di due statue frammentarie di loricato e togato in marmo tasio dall'altezza ricostruita intorno ai $\mathrm{m} \mathrm{2,80} \mathrm{ed} \mathrm{ambedue} \mathrm{dal} \mathrm{retro} \mathrm{non} \mathrm{rifinito} \mathrm{(fig.} 3 \mathrm{~A} \mathrm{e} \mathrm{B}$ ), di una corazza imperiale (fig. $4 \mathrm{~A} \mathrm{e} \mathrm{B}$ ), e alcuni frammenti di incorniciature pertinenti diversi clipei ${ }^{34}$, di una testa ritratto maschile frammentaria (il cosiddetto Traianus Pater, fig. 5 A, B, C, D), rinvenuti all'inizio degli scavi novecenteschi (tra il 1926 e il 1928) nel tratto tra l'angolo dell'aula di testata nord e il Grande Emiciclo, di una testa ritratto femminile rinvenuta nel completare lo scavo dell'area antistante l'aula di testata nord nel 1930 (la cosiddetta Agrippina Minor fig. 6 A, B, C) ${ }^{35}$. Le incorniciature e le teste sono in marmo lunense, quest'ultime ambedue dell'altezza ricostruita $\mathrm{cm} 60 \mathrm{circa}^{36}$. La particolarità di questi rinvenimenti è che si collocano in margine alla fabbrica di dei Mercati di Traiano e non all'interno dell'esedra e/o in margine al portico orientale (fig. 7). Qui non abbiamo notizia di rinvenimenti di statue di «Daci», ma in compenso nell'area di scavo prossima a via di Campo Carleo abbondano i frammenti di pannelli con rappresentazione di armi.

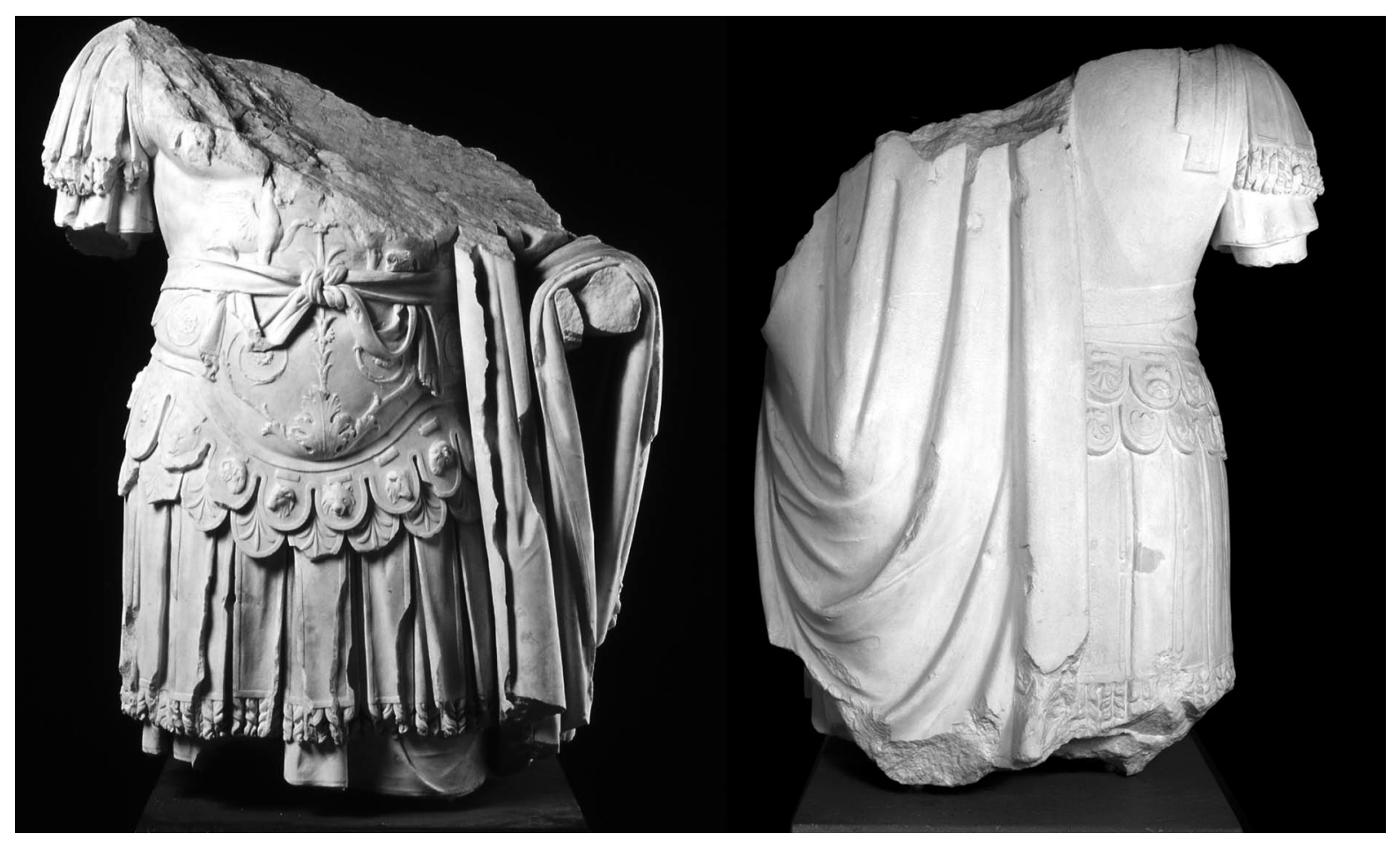

FIG. 3. Foro di Traiano, emiciclo orientale della piazza. Statua frammentaria di personaggio imperiale loricato. Inv. FT 6121. A: visione frontale B: retro non finito.

34 Milella 1995, 236, cat. n. 110.

35 Giornali dei lavori del Governatorato, "Scoprimento del Foro di Traiano", II fascicolo 20/5/192925/5/1930, $1^{\circ}$ maggio 1930 (p. 58): il prezioso materiale d'archivio è conservato presso l'Archivio Storico della Sovrintendenza Capitolina Beni Culturali (SBCAS). Di seguito citazione: cd Agrippina.

36 Ungaro (schede cat.), in Parisi Presicce et al. 2017, 401, n. 16; 438, n. 43. 


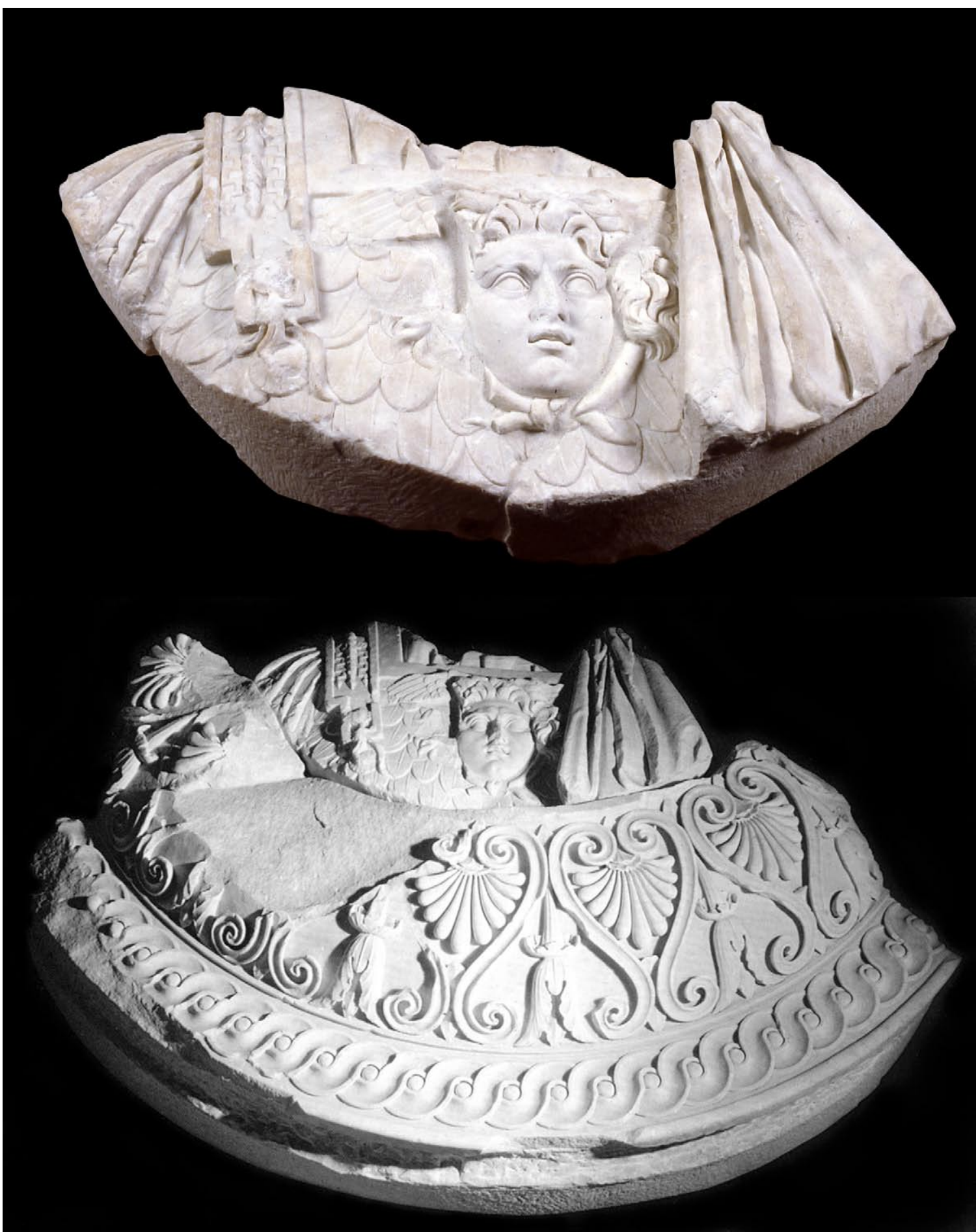

FIG. 4. Foro di Traiano, emiciclo orientale della piazza. A: busto frammentario con corazza imperiale da imago clipeata. Inv. FT 6118. B: allestimento del frammento precedente con l'incorniciatura di un clipeo risultante dall'unione di due frammenti. Inv. FT 4037-4038. 


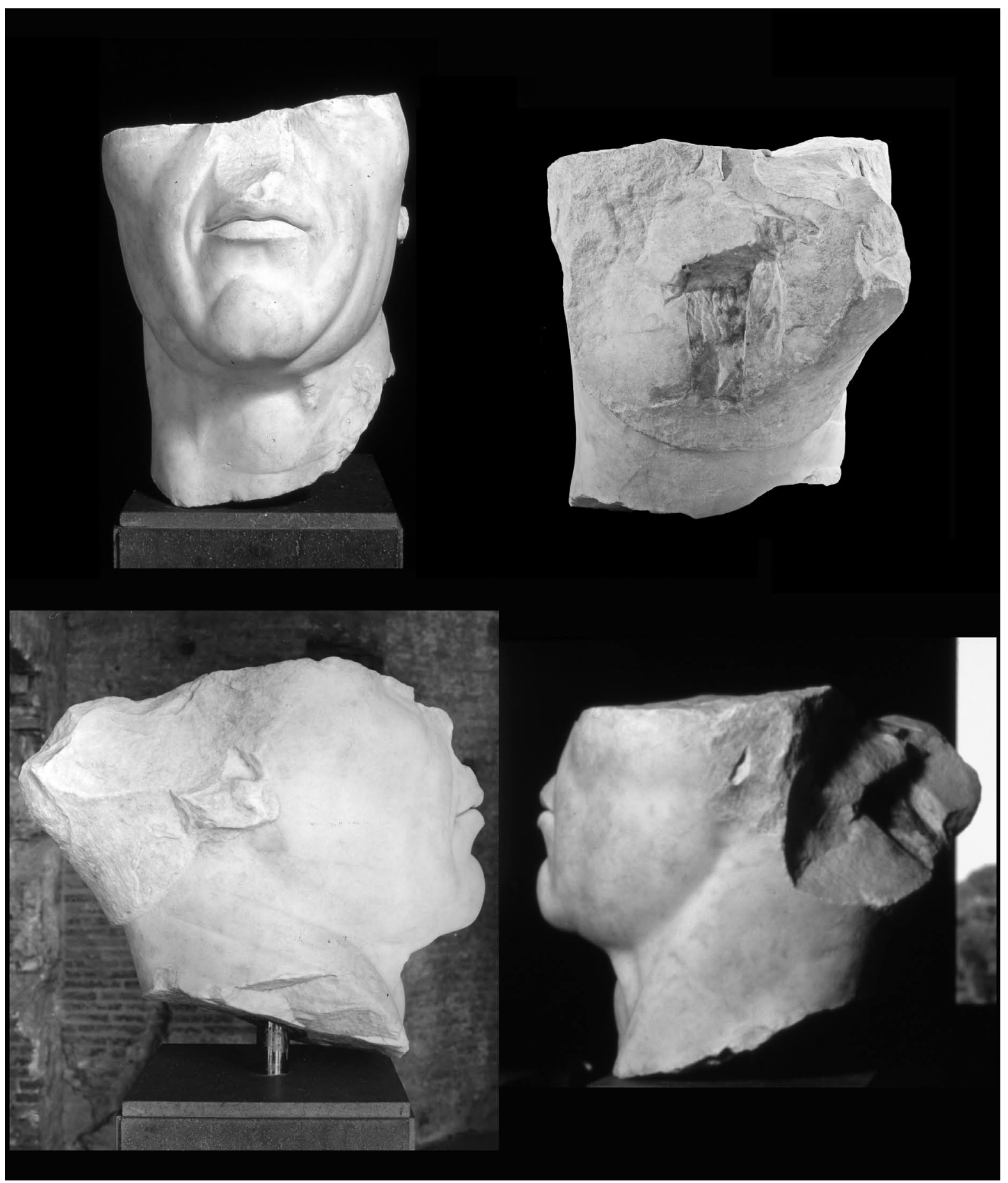

Fig. 5. Foro di Traiano, emiciclo orientale della piazza. Testa ritratto del Traianus Pater. Inv. FT 6117. A: visione frontale. B: retro. C: profilo lato destro. D. profilo lato sinistro. 

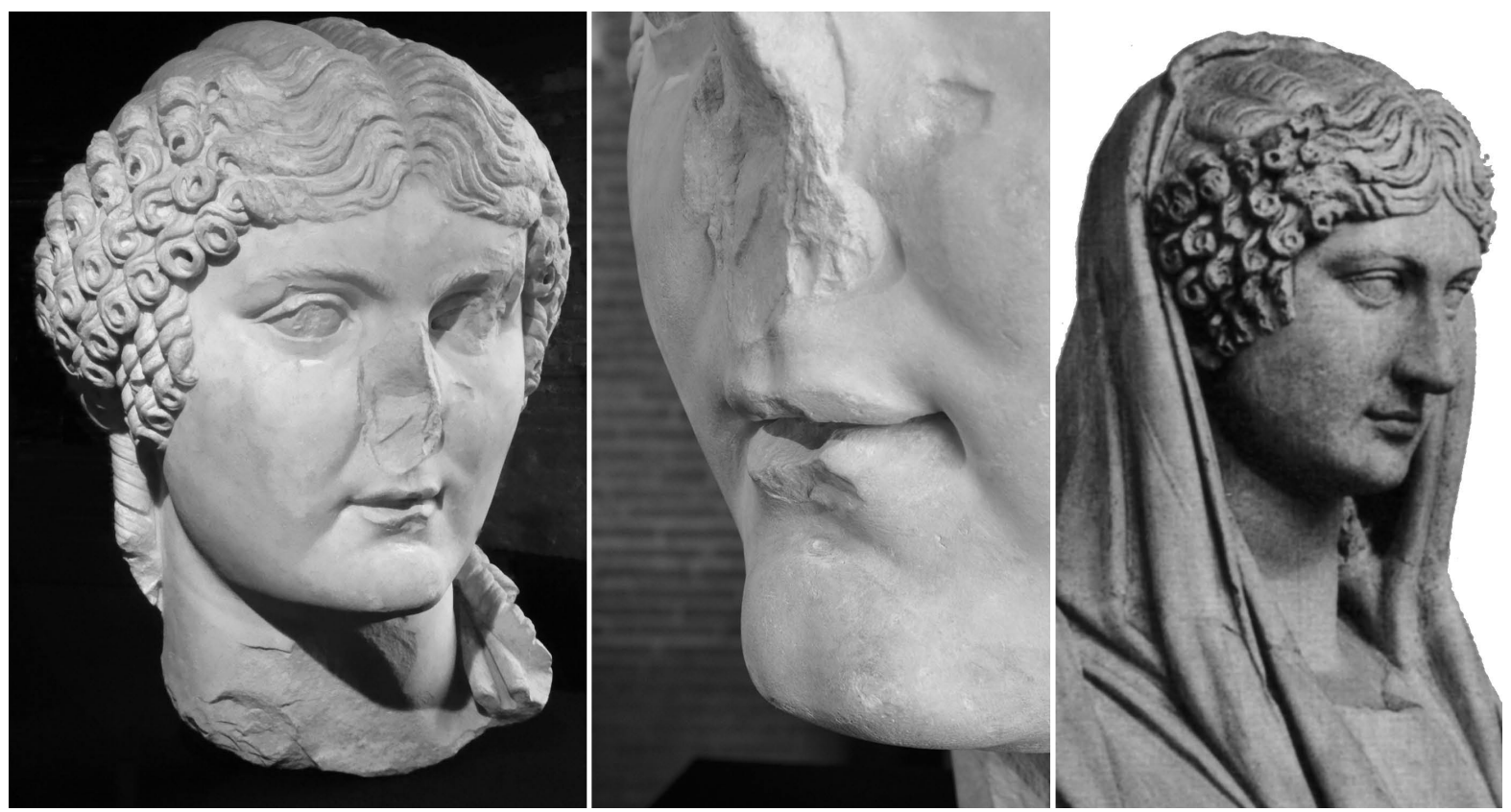

Fig. 6. Foro di Traiano, emiciclo orientale della piazza. Testa ritratto della cosiddetta Agrippina/Marcia. Inv. FT 6116 A: visione frontale. B dettaglio della bocca e del mento. $C$ dettaglio della figura femminile velata dalla Loggia dei Lanzi a Firenze. 


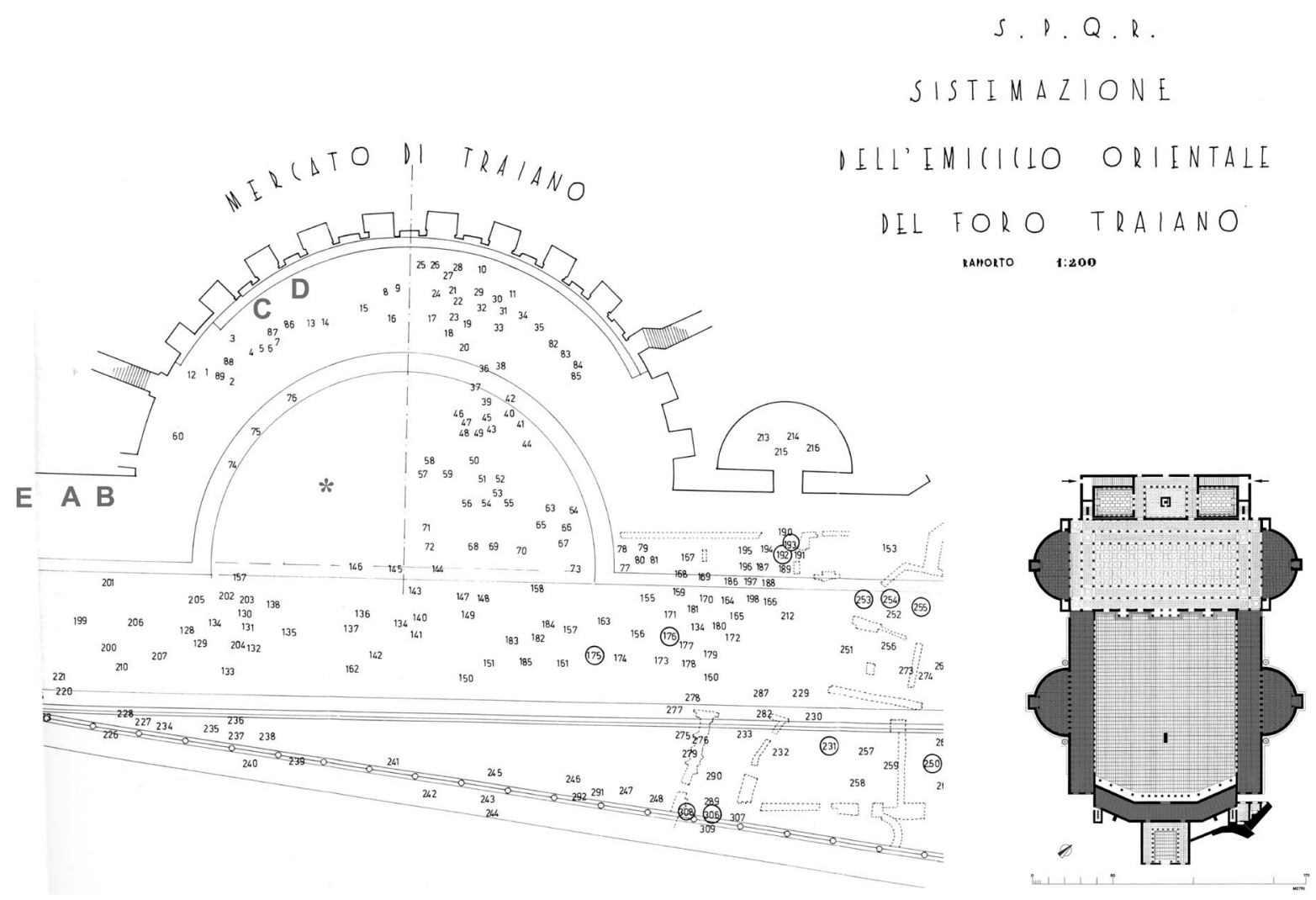

FIG. 7. A sinistra dell'immagine: pianta dell'emiciclo e del portico orientali del Foro, realizzata durante gli scavi del Ventennio del secolo scorso dall'ingegnere G. Cavarra (copia dall'originale Archivio Storico della Sovrintendenza Capitolina Beni Culturali), con l'indicazione dei rinvenimenti scultorei e architettonici; A: statua di loricato (inv. FT 6121); B: statua di togato (inv. FT 6120); C: ritratto di Traianus Pater (inv. FT 6117); D: busto con corazza per clipeo inv. FT 6119; E: ritratto di Agrippina/Marcia (inv. FT 6116). Con asterisco ${ }^{*}$ è indicata l'area di rinvenimento del ritratto di Giulio Cesare. A destra la pianta del Foro di Traiano come in figura 2, con la campitura degli spazi interessati dalla collocazione di sculture.

In quegli stessi anni si scava il Foro di Augusto e si rinvengono incorniciature di clipei recanti al centro teste di Giove Ammone e di barbari, alternati a statue femminili di korai ${ }^{37}$. Gli studi ricostruttivi sono condotti in parallelo dallo stesso studioso, Italo Gismondi, che guida la ricomposizione del partito architettonico con korai e clipei nella Casa dei Cavalieri di Rodi, studia gli edifici augustei, ne mette in cantiere il plastico ricostruttivo della zona scavata. Inevitabilmente, rileva le somiglianze col foro traianeo, per il quale realizza altrettanti rilievi e disegni ricostruttivi: inserisce statue nelle nicchie delle esedre dei portici, clipei sulla facciata dei portici alternati questa volta a statue di Daci, facendo riferimento ai ritrovamenti concentrati in margine al Grande Emiciclo dei Mercati di Traiano ${ }^{38}$.

37 Da ultimo sui lavori al Foro di Augusto e nella Casa dei Cavalieri di Rodi: Ungaro 2016, 281-310.
38 Messa \& Ungaro 1989, figg. 100, 106, 108, 109. 
Successivamente, le due statue e i due ritratti sono conservati nei Mercati di Traiano fino a quando se ne dispone il restauro e l'allestimento ${ }^{39}$. Alcune migliaia di frammenti vengono distesi a terra all'ultimo piano dei Mercati di Traiano e lì restano fino a metà degli anni Ottanta; un certo numero di frammenti di scultura che paiono importanti sono conservati in un ambiente separato. I lavori di restauro e per la nuova destinazione d'uso del monumento imporranno il trasporto in altre aree dello stesso complesso, mentre migliaia di frammenti estratti dall'ulteriore scavo della Basilica Ulpia e della Biblioteca occidentale nel secolo scorso rimarranno nella vasta area sotterranea del monumento, salvata dal reinterro grazie a Corrado Ricci e Amleto Paroli ${ }^{40}$.

Paul Zanker nel 1970 pubblica un articolo fondamentale per i successivi studi sul foro traianeo, nel quale mette in relazione l'emissione di aurei «di restituzione» del 107 d.C. ${ }^{41}$, con il riferimento ai modelli positivi cui Traiano dichiara di ispirarsi —e tra questi Giulio Cesare occupa un posto di primo piano - con i ritratti noti e di provenienza presumibile dal Foro; riprende la proposta del Gismondi sui clipei; alimenta l'ipotesi della relazione tra il ritratto della cd Agrippina con la statua femminile appartenente alla serie delle «Sabine» oggi esposte nella Loggia dei Lanzi a Firenze.

Ricordiamo brevemente che in questo ciclo statuario unitario si riconoscono ritratti di personaggi femminili importanti della famiglia imperiale - Marciana, Matidia Maggiore, Sabina con testa ideale - la cosidetta Thusnelda, una donna «barbara», con l'aggiunta della cd Agrippina, e statue ideali pertinenti summae mulieres. Le imponenti figure femminili in media alte $\mathrm{m} 2,60$ sono attestate nella collezione raccolta nel palazzo Della Valle fin dal 1532 e il 1541 (la cd Agrippina) ${ }^{42}$, confluiscono nella collezione Della Valle-Capranica, vengono acquistate da Ferdinando de' Medici e collocate nella villa al Pincio, dove rimangono fino al trasferimento a Firenze per l'esposizione nella Loggia dei Lanzi dal 1787. La matrona velata, ritratta in stile giulio-claudio, viene identificata con Agrippina Minore ${ }^{43}$, o variamente interpretata come una delle Agrippine ${ }^{4}$.

In tempi recenti è stato eseguito un nuovo intervento di restauro delle statue ${ }^{45}$, che ne ha circoscritto la datazione alla tarda età traianea o primo adrianea, accertando per le figure femminili di rango imperiale l'impiego di marmo tasio (dell'area di Saliara, prossima all'area di Vathy), come le due statue maschili di loricato e togato, mentre per la Thusnelda, la figura di barbara spesso ritenuta addirittura non antica, l'esecuzione in marmo frigio bianco dall'area delle cave di Altintas, prossime ad Afyon (Docimium) ${ }^{46}$. Il ciclo unitario familiare, vista anche l'altezza media di m 2,60, ben si adatterebbe alle esedre della Basilica Ulpia. Non deve stupire l'inserimento della nipote di

39 Ungaro 1993, 129-174: fu eseguito nel 1982 dopo il restauro ad opera di Cecilia Bernardini, su progetto di Paolo Martellotti sotto la direzione di Eugenio La Rocca; la collocazione era nella sala absidata del primo piano del Corpo Centrale dei Mercati di Traiano. Nel 1995 fu realizzata la prima mostra sui materiali restaurati dai Fori di Augusto e di Traiano: Ungaro \& Milella 1995.

40 Ungaro 2005, 69-86. Tutt'oggi l'area sotterranea è di straordinaria importanza per le emergenze archeologiche (la pavimentazione originaria, i crolli in situ delle strutture, resti di epoche successive, ecc.) e per la quantità di reperti conservati, alcuni dei quali fondamentali per la conoscenza del Foro. Sono stati fatti vari progetti di recupero e valorizzazione, per ora privi di adeguato finanziamento.

41 Zanker 1970, 499-544 (518-521); Woytek 2010, 641-644.

42 In questa collezione sono presenti reperti importantissimi provenienti o attribuibili al Foro, come vedremo oltre.

43 Mansuelli 1958-1961, II, n. 59.

44 Capecchi 1975, 169-178; Gasparri 1979, 524-543.

45 Sotto il coordinamento di Romualdi $2002 \mathrm{a}$, 17-18, 26-27.

46 Ambedue le cave dell'Asia Minore facevano capo alla gestione amministrativa di Synnada, e producevano marmi colorati e bianchi. 
Traiano, in una galleria di questo genere, anche se Matidia nel 112 d.C. (all'atto dell'inaugurazione del Foro) non è ancora elevata ad Augusta ${ }^{47}$.

Nel catalogo dei ritratti dei Musei Capitolini, per la testa romana Paul Zanker conferma l'ispirazione del ritratto ad Agrippina Minore ma con caratteristiche tipiche della lavorazione traianea: un ritratto postumo? ${ }^{48}$. Rimane però la difficoltà di spiegare la presenza di una così discussa donna giulio-claudia nel ciclo forense: gli studiosi Eck e Boschung ${ }^{49}$ propongono di identificare nella nostra testa, e quindi anche nella testa velata della Loggia, la madre di Traiano, non attestata dalle fonti, tanto che non se ne conosce il nome ma lo si desume da quello della ben nota figlia, Marciana, come Marcia. La futura moglie dell'imperatore doveva essere esponente di una famiglia importante, è stata identificata nella proprietaria di importanti figlinae nell'area umbra, ma sempre per la continuità di presenza con la figlia ${ }^{50}$. Secondo l'ipotesi Eck e Boschung, Marcia sarebbe raffigurata con l'acconciatura della sua epoca giovanile, appunto ispirata ad Agrippina Minore, ma con caratteristiche della lavorazione di età traianea e con tratti del volto diversi, come l'ovale del viso più squadrato, la bocca piccola, il mento pronunciato ${ }^{51}$ (cfr. fig. 6).

Sul disconoscimento dell'identificazione con l'esponente giulio-claudia bisogna considerare anche un diverso punto di vista. La Wood infatti nella sua monografia rileva come gli storici romani abbiano decisamente dipinto in negativo la futura moglie di Claudio, dimenticando l'autentica capacità di governo dimostrata dall'unica donna-principe che abbia governato da sola per cinque $a_{n} i^{52}$. La conferma viene anche dalla quantità di tributi statuari che ci restano di Agrippina Minore, davvero impressionante. Il ritratto postumo dal Foro si inquadrerebbe nella serie di ritratti di donne e uomini delle dinastie precedenti e segnerebbe una precisa volontà di Traiano di differenziarsi dal Nerone della fase ultima e buia, di dimostrare la diversa considerazione che lui ha per le figure femminili ${ }^{53}$. All'inizio del II sec. d.C. in effetti, erano trascorsi alcuni decenni, ma erano ancora visibili statue di Agrippina Minore e il popolo poteva aver ormai dimenticato la sua fine innaturale.

Stride con questa lettura il fatto che avremmo rinvenuto per ben due volte la rappresentazione dello stesso personaggio (nella testa colossale e nella statua dalla Loggia) a fronte dalla scarsa presenza almeno per ora di altre figure femminili (a parte quelle della serie della Loggia).

Nell'ambito delle opere di sicura provenienza dal Foro ${ }^{54}$, rientra il ritratto di Giulio Cesare oggi esposto al Museo Archeologico Nazionale di Napoli, col quale torniamo ai rinvenimenti dall'emiciclo

47 Scheithauer 2000, 154-166; di diverso parere Kreikenbom 1992, 205.

48 Fittschen \& Zanker 1985, 6, n. 5, tav. 6.

49 Boschung \& Eck 1998, 473-481.

50 Champlin 1983, 257-264; Charles-Laforge 2015, 233-273. Chausson 2007, supra nota 4.

51 Vedi in seguito il confronto con i tratti del volto di Traiano.

52 Wood 1999, 257-259, 295-305: esamina il contesto della "corte» imperiale e la copiosa quantità di ritratti pervenuti. Cfr. anche Rose 1997, 69-70. E' vero che Agrippina che non venne mai riabilitata dal Senato, ma aveva ricevuto il titolo di Augusta e le popolazioni germaniche riconoscevano in lei le capacità di un vero leader.

53 Wood 1999, 314: secondo l'A., al contrario, la scomparsa delle donne dall'arte pubblica di Nerone è sintomatica del collasso della dinastia.

\footnotetext{
54 In questa sede non affrontiamo i ritratti oggi nella collezione Villa Albani-Torlonia (Ungaro 2017 a, 94), benché di grande interesse: il ritratto colossale di Traiano presenta infatti misure compatibili con le nostre teste (altezza capo mento di $\mathrm{cm} \mathrm{60),} \mathrm{è} \mathrm{rivolta}$ a sinistra, ma è del tipo precedente a quello del decennale e, quindi, sarebbe un po' anomalo il suo inserimento nella galleria del Foro di Traiano, ma anche questo esemplare dovrebbe provenire, insieme al noto pannello con amorino dal Tempio di Venere Genitrice, dalla collezione nel palazzo Della Valle-Capranica transitata in quella Medici nella villa del Pincio: cfr. Amedick (scheda cat.), in Bol 1994, 471-472, n. 948; Milella 2007, 111, fig. 130: per l'esposizione nel Museo dei Fori Imperiali di un bel frammento proveniente dagli scavi del secolo scorso del Foro di Cesare. Per la possibile provenienza Gasparri 2007, 79 e nota 46: l'Autore associa in via ipotetica anche il ri-
} 
orientale del Foro di Traiano (fig. 8 A, B). Infatti, il suo ritrovamento nell'area Spoglia Christi è certo $^{55}$, sappiamo che proviene dallo scavo nel giardino della famiglia Guccini, chiaramente evidente anche oggi perché corrisponde alla superficie priva di resti della pavimentazione marmorea originaria.

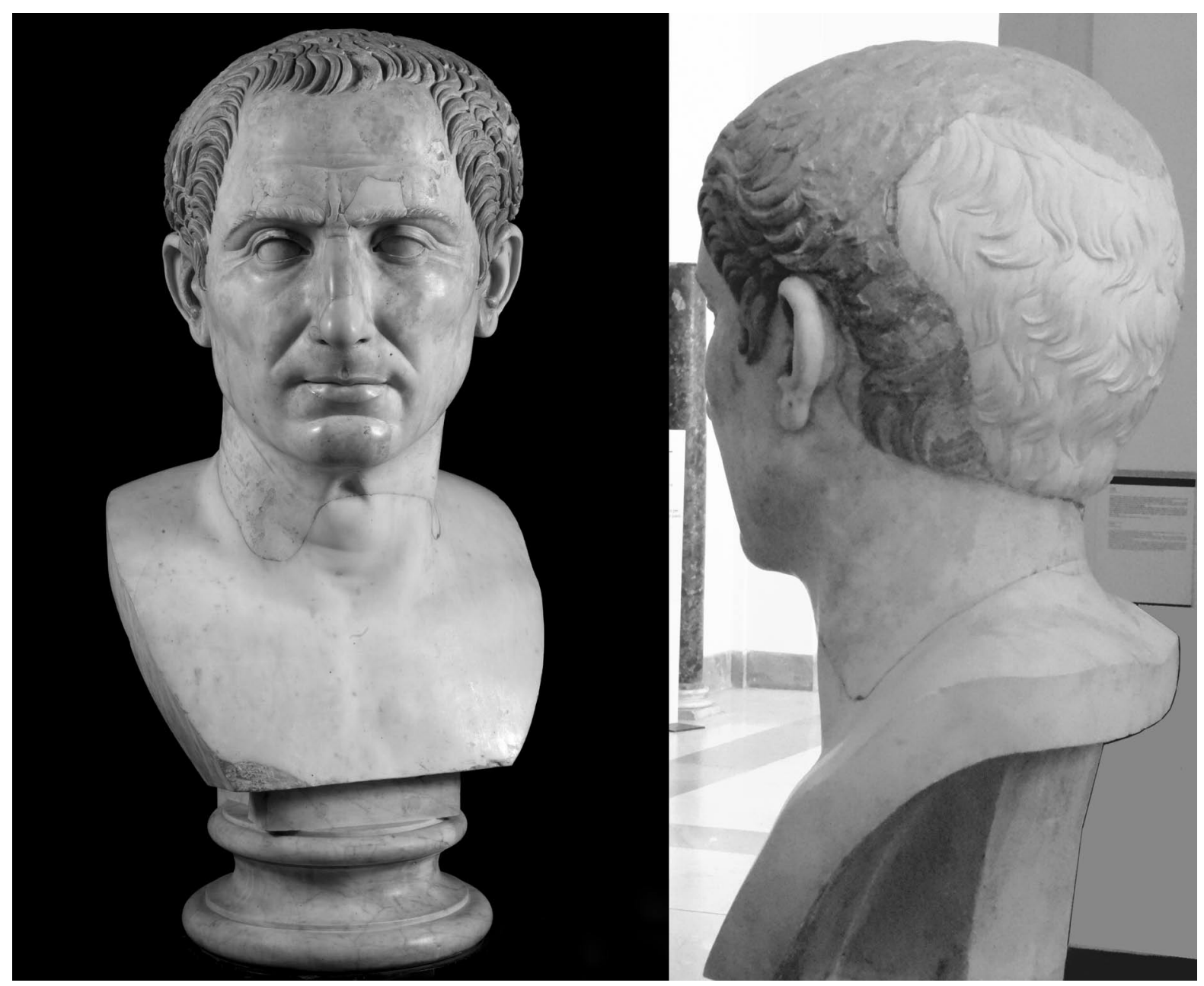

Fig. 8. Ritratto di Giulio Cesare dalla Collezione Farnese. Museo Archeologico Nazionale di Napoli. A: visione frontale b: retro con zona occipitale integrata.

L'importante ritratto della Collezione Farnese è stato ricondotto ad una rielaborazione in età traianea di un tipo creato poco prima della morte di Giulio Cesare ${ }^{56}$, come anche quello apparte-

tratto di Tito (da lui definito di Vespasiano), ma questo ha un'altezza di ben $\mathrm{cm} 68$ (capo-mento): Amedick (scheda cat.) in Bol 1994, 470-471, 947. Importanti i riscontri in Gross 1940, 54-90 (per la classificazione tipologica); 124, scheda cat. 4 e tav. 4 (busto da Villa Albani-Torlonia); 129, scheda cat. 42 e tav. 14 c,d (ri- tratto su statua loricata non pertinente da Villa Albani-Torlonia).

55 Grazie ai puntuali studi archivistici di Meneghini 1992, 409-436.

56 Si veda Coraggio (scheda cat.) in Gasparri 2010, 62-63, n. 37, tav. XXXVI, 1-5. 
nente alla statua ritratto colossale di Palazzo Senatorio, che è strettamente connessa alle nostre statue di loricato e togato e sembra condividerne l'origine ${ }^{57}$. La zona occipitale della testa presenta una vasta integrazione, ma non sappiamo dovuta a quali possibili cause. Le misure sono compa-

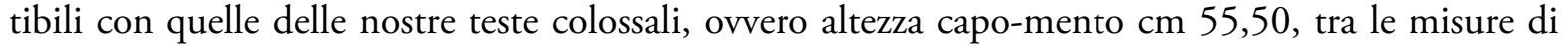
dettaglio la bocca è $\mathrm{cm} \mathrm{10,5/11.} \mathrm{Anche} \mathrm{in} \mathrm{questo} \mathrm{caso,} \mathrm{come} \mathrm{in} \mathrm{quello} \mathrm{del} \mathrm{personaggio} \mathrm{femminile,}$ lo stesso personaggio sarebbe rappresentato due volte evidentemente in contesti diversi del Foro.

In sintesi, le due statue di loricato e togato e il Giulio Cesare di Palazzo Senatorio presentano un'altezza ricostruita compatibile con le figure femminili della Loggia, facendo pensare quindi a possibili gallerie di sculture, a cicli con finalità diverse ma tutto programmato con l'obiettivo di rafforzare l'immagine di Traiano, ispirandosi alle virtù dei migliori prima di lui e ai valori della famiglia, nel contesto di un'opera publica per eccellenza, espressione della maiestas imperii romani ${ }^{58}$.

Passando ai ritratti, le proporzioni sono completamente diverse rispetto alle statue della Loggia e a quelle dal Foro e questo dato viene avvalorato oltre che dalle tre teste del Traianus Pater, dell' "Agrippina-Marcia» e del Giulio Cesare, da preziosi frammenti recentemente ripresi in considerazione.

Durante il riordino di frammenti di scultura attualmente in deposito presso la Basilica Ulpia, ma in origine nell'ambiente riservato all'ultimo piano dei Mercati ${ }^{59}$ ne sono riemersi già noti e non noti che fortunatamente sono stati ricongiunti.

Un volto frammentario è quello già pubblicato nel catalogo dei Musei Capitolini da noi ritenuto maschile giovanile e non femminile: sviluppa $\mathrm{cm} 55$ di altezza per una statua di almeno $\mathrm{m}$ 4,50: la bocca ricorda molto da vicino quella del Traianus Pater ${ }^{60}$.

Un altro frammento pubblicato sempre nello stesso catalogo è limitato al solo occhio destro: oggi fortunatamente è stato ricongiunto ad un altro reperto che conserva in parte l'orecchio destro e la terminazione della capigliatura sulla tempia con una basetta ${ }^{61}$ : è stato agevolmente possibile risalire al ritratto di Traiano tipo decennalia con uno sviluppo in altezza di circa $\mathrm{cm} 60^{62}$, quindi, siamo sempre in presenza di una testa ritratto colossale per una statua di m 4,80 circa, oppure di un ritratto per clipei eventualmente in analogia al Traianus Pater (fig. 9 A, B). Infatti, le statue del resto, sarebbero sì colossali ma ben proporzionate alle misure dei giganteschi ordini architettonici del Foro.

\footnotetext{
57 Albertoni 1993, 175-183; Ungaro (scheda cat.), in Parisi Presicce et al. 2017, 391-392, n. 6.

58 Importante in questo contesto lo studio in corso da parte di Milella 2017, 192-211 e (scheda cat.) in Parisi Presicce et al. 2017, 430-431, n. 33. su una mano frammentaria pertinente una statua colossale maschile imperiale, per la quale è stata avanzata la proposta di collocazione in una delle nicchie centrali delle absidi basilicali.

59 Il dettaglio non è irrilevante per stabilire la provenienza almeno ipotetica dei reperti, ricordando però che nell'area di Campo Carleo esisteva una fornace molto attiva in età altomedioevale e che, quindi, molto materiale può essere stato apposita-
}

mente «ammassato» in questa zona per essere ridotto in calce.

${ }^{60}$ Fittschen \& Zanker 1985, I, n. 38, tav. 40; Ungaro (scheda cat.) in Parisi Presicce et al. 2017, 440, n. 45 .

${ }^{61}$ Ungaro (scheda cat.) in Parisi Presicce et al. 2017, 439-440, n.44. La «riemersione» dei frammenti si deve alla collega Marina Milella durante lavori di sistemazione del deposito, mentre il provvidenziale «attacco» tra i due frammenti si deve al collega Simone Pastor: ringrazio ambedue.

${ }_{62}$ Grazie al suggerimento di Eugenio La Rocca e all'abilità di Maria Luisa Vitali nella ricomposizione grafica. 

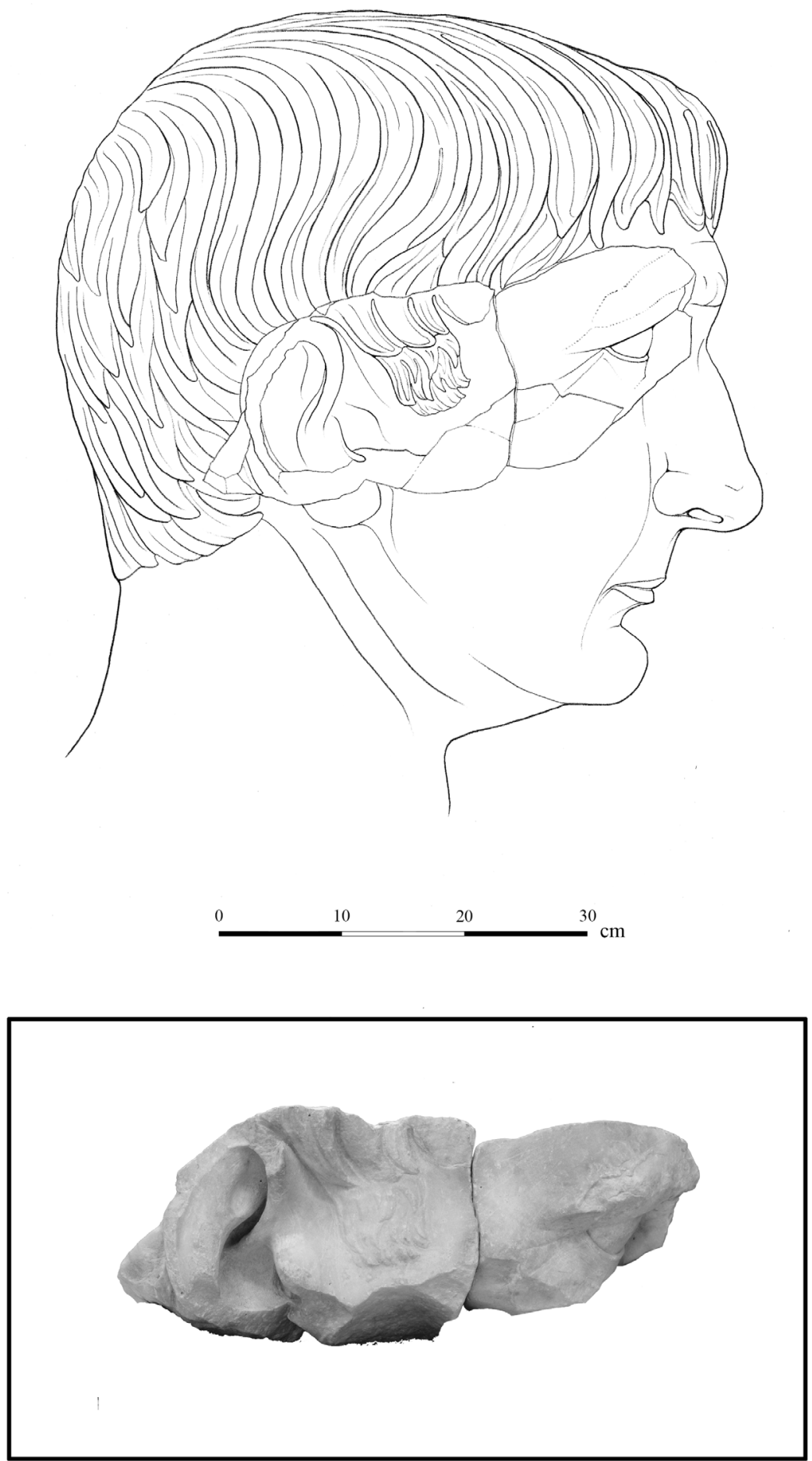

Fig. 9. Ritratto colossale di Traiano tipo decennalia. A. ricomposizione frammenti inv. FT 8720 (con occhio), FT 85 (con orecchio). B disegno ricostruttivo (a cura di M. L. Vitali). 
Il nostro nuovo ritratto presenta dettagli coincidenti con quello dei decennalia: malgrado l'esiguità dei due frammenti, si riconosce l'età matura dal trattamento degli occhi con le rughette, l'occhio stretto allungato, le palpebre sottili, la borsa sotto l'occhio appena accennata; il padiglione auricolare ben caratterizzato ${ }^{63}$ (cfr. fig $1 \mathrm{~B}$ ). La capigliatura con terminazione delle ciocche lunghe e sottili sulla tempia, la basetta con taglio rettilineo netto corrisponde alle caratteristiche del tipo IV del ritratto imperiale come ben definito nella monografia di Gross ${ }^{64}$ e nella dettagliatissima classificazione delle emissioni monetali traianee di Woytek ${ }^{65}$.

Sia nel caso di Traianus Pater sia in quello di «Agrippina, Marcia», le tracce nella zona occipitale delle due teste mostrano come il perno ossidandosi e "saltando» violentemente dalla sua sede (o per evento traumatico o per altro) abbia causato la frantumazione del marmo tutto intorno con distacco di frammenti consistenti ${ }^{66}$. Lo stesso destino potrebbe essere toccato al Giulio Cesare poi abilmente integrato.

Mentre nel caso dell' "Agrippina, Marcia», il foro praticato e la rifinitura della capigliatura sul retro fanno propendere per l'ipotesi di una statua colossale dedicata alla madre dell'imperatore ${ }^{67}$, nel caso del Traianus Pater persiste la possibilità dell'inserimento in un clipeo come proposto in passato, anche se a nostro avviso la conformazione anomala della nuca, non rifinita sul retro, non fa comprendere come dovrebbe essere agganciata la pesante testa alla lastra di fondo (cfr. fig. 5 C, D). Inoltre, la relazione con il busto loricato ritagliato in modo tale da essere certamente inserito in un clipeo può funzionare per le proporzioni, ma il personaggio rappresentato indossa una corazza squamata sulla quale campeggia la gorgone minacciosa ed è caratterizzata dalla folgore di Giove sugli spallacci, segno di comando imperiale; inoltre, lo scollo è quadrato, ovvero la lorica potrebbe essere già adrianea (cfr. figg. 4 e 11$)^{68}$. Si dibatte sulla data della divinizzazione del padre genetico dell'imperatore: infatti, il Traianus Pater è definito Divus in due diverse serie monetali del 112/113 d.C. secondo la classificazione fatta da Woytek, associato a Nerva e da solo ${ }^{69}$. Ma sulla data della divinizzazione è stato notato che un evento così significativo non viene menzionato nei Fasti Ostiensi del 112/113 d.C. pervenuti, pertanto, viene proposto di collocare la divinizzazione nel periodo del quale non ci sono pervenuti i Fasti Ostiensi, tra maggio 113 e agosto 114 d.C. ${ }^{70}$. In questo caso dovremmo pensare ad un clipeo realizzato e posto in opera in un settore/edificio non inaugurato nel $112 \mathrm{~d}$.C. ma inserito nelle aree monumentali terminate per ultime: o l'area sud

\footnotetext{
63 Colugnati (scheda cat.) in La Rocca, Parisi Presicce \& Lo Monaco 2011, 261, cat. I, 3.

64 Gross 1940, 85-90, 129, scheda cat. 41, tav. 15 b.

65 Woytek 2010, 608, n. 212b, tav.43 (consolato $\mathrm{V}$; datazione $106 / 107$ d.C.; ritratto tipo B); 609, n. 224 e, tav.45 (consolato V; datazione 107 d.C.; ritratto tipo B/C; sul retro corona civica e legenda OPTIMI PRINCIPI); 611, gruppo 10 n. 261 f, r-270 a, b, c, d.

66 Da notare che questi dettagli nella lavorazione delle teste possono essere spia di un particolare "montaggio", come se fossero composte da più parti e portassero perni metallici per essere ancorate meglio a "pareti» retrostanti, in pratica applicando la tecnica dell'acrolito ma a statue presumibilmente tutte in marmo "fatte a pezzi», oppure a clipei. In quest'ultimo caso occorre un maggiore approfondimento sul sistema di aggancio e sulla valutazione del
}

peso delle teste che dovevano essere leggermente inclinate verso il basso.

67 Anche perché non vi sono casi conosciuti di imagines clipeatae in luoghi pubblici dedicate a figure femminili. Si veda la dettagliata rassegna di Winkes 1969.

68 Per la corazza n. inv. FT 6119: Ungaro 1993, 166, fig. 90; Ungaro 1995, 130, n. 61.

69 Woytek 2010, 622, n. 400f, tav.84; nn. 405f, $406 \mathrm{f}(2)$, tav.85 (consolato VI; datazione 112/113 d.C.; legenda sul retro: DIVI NERVA ET TRAIANUS PATER, quindi i due padri compaiono ambedue divinizzati; Nerva lo era già stato nel 98 d.C.); nn. 401 a, $401 \mathrm{~b}(1), 401 \mathrm{~b}(2), 402 \mathrm{v}$, tav. 84; $406 \mathrm{~b}(1), 406 \mathrm{~b}(2)$, $407 \mathrm{f}, 408 \mathrm{f}(1), 408 \mathrm{f}(2)$, tav.85 (consolato VI, datazione 112/113 d.C.; legenda sul retro DIVUS PATER TRAIANI con chiaro e inequivocabile riferimento alla divinizzazione di Traianus Pater, che compare da solo).

70 Durry 1965, 48-53, ripreso da Alföldi 1998, 12. 
con l'aula trisegmentata, o l'area nord, dove ancora è aperto il dibattito sul tempio. Ricordiamo che la moneta con l'immagine attribuita alla facciata dell'aula trisegmentata, ma che potrebbe essere letta anche come la rappresentazione di un arco monumentale, presenta un fornice centrale e due nicchie per lato, sovrastate da clipei, per un totale di cinque elementi, e una statua inserita in ogni nicchia per un totale di quattro elementi ${ }^{71}$.

In ogni caso il riconoscimento del nuovo ritratto colossale di Traiano del tipo decennalia della stessa misura o molto prossima (tra 55 e $60 \mathrm{~cm}$ ) a quella di altri ritratti può suggerire una "galleria» dedicata alla famiglia (padre genetico e madre) e ai modelli (come Giulio Cesare) di grandi proporzioni (mediamente $\mathrm{m} 4,80$ ). Il luogo di rinvenimento di questi esemplari sembra concentrarli nell'emiciclo e nel portico orientale (anche se gli scavi non hanno rilevato tracce in fondazione per sostenere basamenti e statue così grandi, ma questo dato non può essere ritenuto definitivo. In alternativa i ritratti maschili potrebbero appartenere ai clipei in facciata dei portici, con le osservazioni fatte sopra per il Traianus Pater.

D'altro canto, abbiamo visto statue che paiono ragionevolmente appartenere al Foro: il ciclo

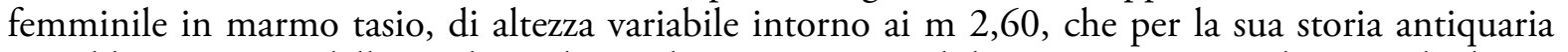
potrebbe provenire dalla Basilica Ulpia, e le tre statue maschili in marmo tasio e lunense di altezza

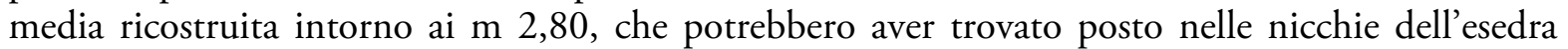
orientale e che fanno pensare ad un ciclo dedicato agli uomini illustri, ai comandanti delle truppe, ai modelli di riferimento.

Sicuramente si dovrà ancora lavorare sul riesame di altri frammenti di sculture presenti in deposito $^{72}$.

Le diverse serie di statue e ritratti devono essere state proporzionate e in coerente relazione con gli edifici, in quanto facenti parte di un unico programma architettonico-scultoreo.

L'altro parametro-guida del programma era certamente il sistema gerarchico tra la rappresentazione dei vinti e dei vincitori: da quello che finora conosciamo dell'apparato figurativo, questo rapporto deve essere stato molto «sensibile» soprattutto nelle facciate degli edifici affacciati sulla piazza, vera materializzazione del trionfo di marmo.

In questo contributo per l'esame delle statue di Daci e di altre popolazioni, e dei pannelli con armi in rapporto alla Colonna Traiana e al basamento della statua equestre si rimanda a quanto già pubblicato $^{73}$, ma si ricordano solo alcuni dati per mettere in relazione tutti i tasselli del complesso apparato iconografico. Le statue dei «vinti» sono tutte colossali, alte tra i m 2,60 e i m 3,00 circa e sono rispettivamente così distribuite:

71 Cfr. supra nota 30.

72 Ad esempio, un altro resto di capigliatura femminile con larghe ciocche molto simile ma non pertinente la capigliatura di Agrippina-Marcia, sembra riportare sempre ad acconciature giulio-claudie: è la spia della presenza di un'altra figura femminile di quella dinastia? Ungaro 2017 a, 96, fig.8 (inv. n. FT 460). La revisione di tutto il materiale scultoreo attribuibile al Foro di Traiano è programmato per il prossimo biennio e finalizzato ad una pubblicazione complessiva.

73 Ungaro 2017 b, 146-150; Ungaro 2017 c, 291296: con bibliografia precedente; Ungaro (schede cat.) in Parisi Presicce et al. 2017, 406-413, nn. 18 a-19 f; 458-461, nn. 60 a-h con bibliografia specifica. 

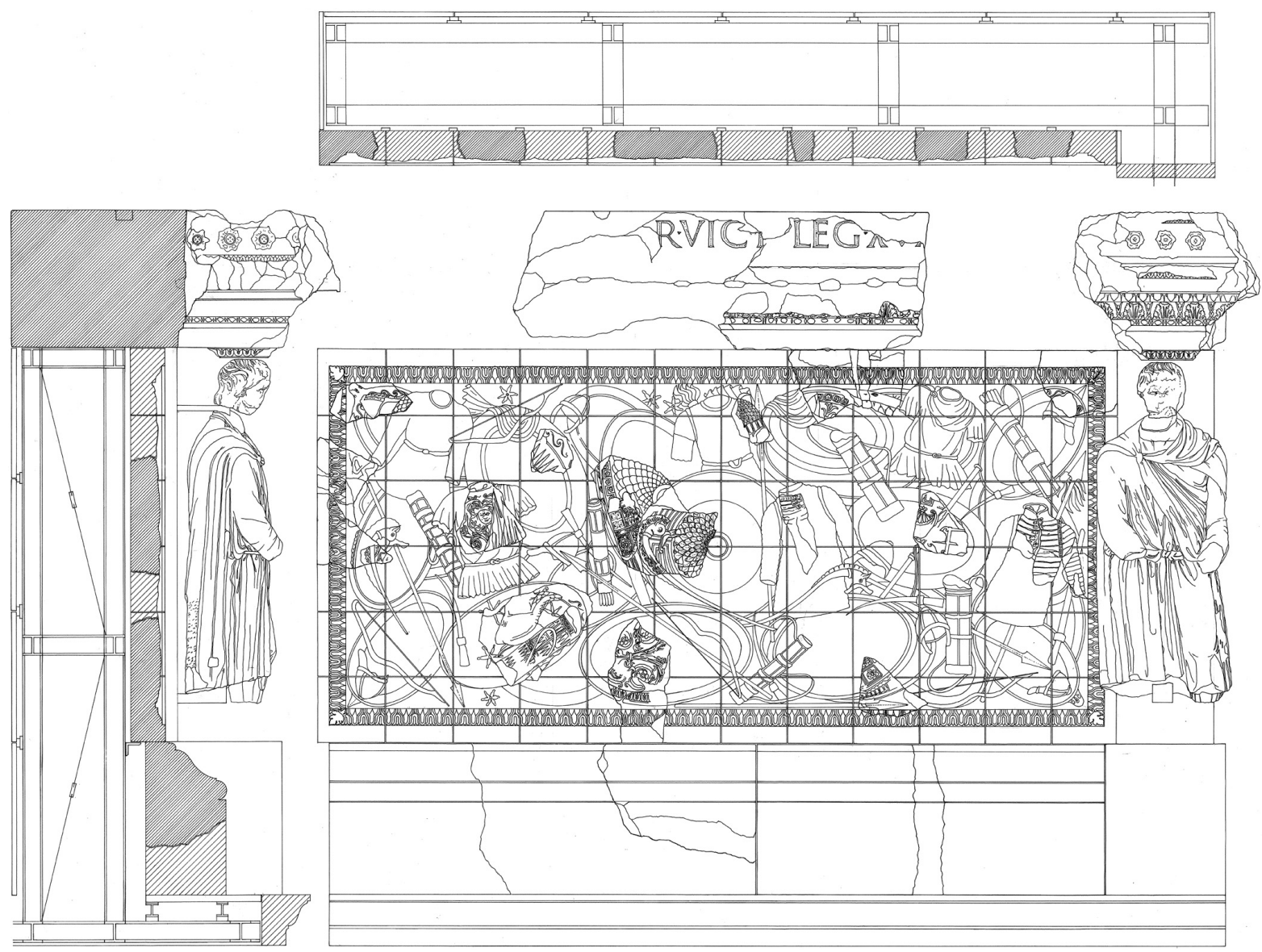

FIG. Io. Basilica Ulpia, facciata sulla piazza, ricomposizione decorazione dell'attico. Statua di dace in marmo bianco inv. FT 6108; pannello ricomposto da frammenti di incorniciatura e di rilievo con armi; elementi di trabeazione sporgente: mensola inv. FT 2584, parte lineare iscritta inv. FT 2658.

— quelle da m 2,60 in marmo bianco sull'attico della facciata della Basilica Ulpia in alternanza ai pannelli con armi e ed in presenza di statue imperiali tra i m 2,70/3,00 sui basamenti iscritti con dedica all'imperatore poste agli ingressi sottostanti dell'edificio ${ }^{74}$; la Basilica è costruita ex manubiis ovvero con i proventi delle guerre daciche e l'attico è completato dalle iscrizioni e dalle insegne delle legioni ${ }^{75}$ (fig. 10);

74 Ungaro 2010, 105-113.

75 Pastor 2017, 286-290. 


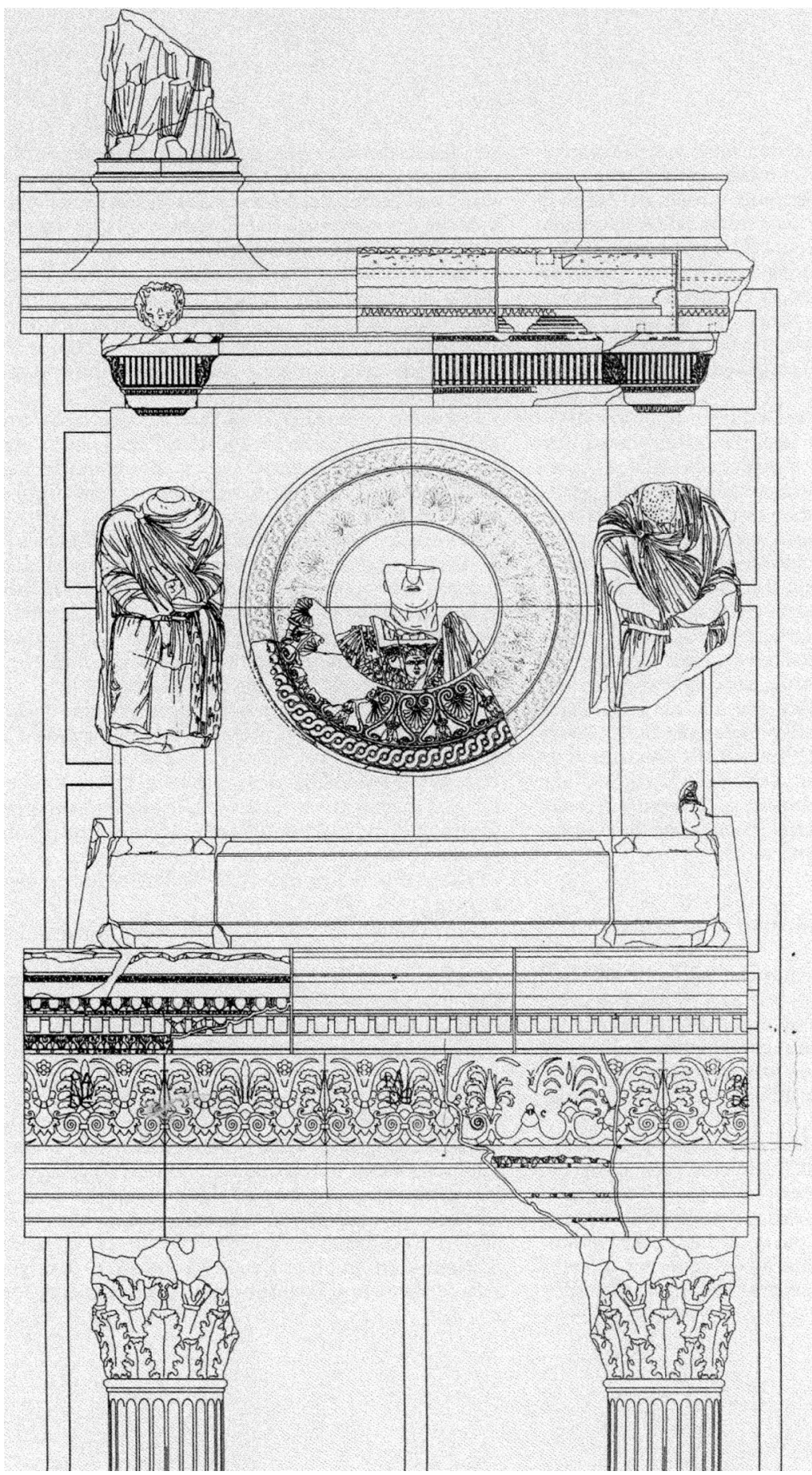

FIg. I I. Portici della piazza del Foro, ricomposizione di un tratto dell'attico: alternanza statue colossali di Daci in marmo bianco inv. FT 6103 e in marmo pavonazzetto inv. FT 6101; imago clipeata composta da busto corazzato inv. FT 6118 e incorniciatura inv. FT 4037-4038. 
— quelle da m 3 ca in marmi bianchi e colorati, veramente imponenti, sull'attico delle facciate dei portici: sono alternate ai clipei contenenti ritratti di personaggi illustri e comandanti militari connessi sempre alle campagne daciche ${ }^{76}$. I clipei misurano m 2,50 di diametro e con la probabile incorniciatura del pannello dovevano arrivare all'altezza dei Daci ${ }^{77}$ (fig. 11);

- due statue alte circa m 2,60 in marmo bianco provengono dallo scavo antistante la facciata dell'aula trisegmentata a chiusura della piazza a sud: se la rappresentazione sulla famosa moneta con l'iscrizione sull'esergo FORUM TRAIAN(I) ritraesse effettivamente questa facciata, sarebbero inserite in un apparato decorativo con clipei e statue, ma, come già accennato, non si può escludere al contrario che la moneta si riferisca all'apparato del lato nord dietro la Colonna Traiana ${ }^{78}$. Di certo, la posizione dell'imponente monumento equestre spostato verso sud ci fa immaginare comunque una facciata monumentale in funzione di sua quinta scenografica.

La piazza smisurata del Foro di Traiano, quindi, col suo programma figurativo incentrato sulla vittoria dell'esercito romano e del suo comandante supremo, la presenza delle insegne e dei ritratti riecheggia sempre più da vicino la funzione dei principia, cuore degli accampamenti militari romani.

Alle tipologie di statue di «vinti» finora viste, dobbiamo aggiungere quelle superlative realizzate in porfido, la pietra imperiale per eccellenza che potrebbero essere associate alla porticus porphiretica. Ricordiamo solo a titolo esemplificativo l'esemplare in porfido rosso oggi conservato presso l'Opificio delle pietre dure, giunto a Firenze sempre attraverso i Medici dalla Collezione Della Valle-Capranica dall'altezza ricostruibile intorno ai $3 \mathrm{~m}$ circa insieme ad altri analoghi ${ }^{79}$. Due piccoli ma significativi frammenti di statue di daci in porfido rosso sono conservati nel deposito del $\mathrm{Mu}$ seo dei Fori Imperiali presso la Basilica Ulpia, uno con la terminazione tipica dei mantelli dacici con frangia (fig. 12,7) ${ }^{80}$. Si conservano inoltre sei frammenti da iscrizioni presumibilmente monumentali in porfido; tra questi uno conserva su due righe le lettere - CV-- e forse -DIV- ma vi è una notevole differenza di esecuzione tra le lettere della prima riga (ben delineate e regolari) e quelle della seconda (fig. 12,1$)^{81}$. Per qualità della resa e per lo spessore della lastra si avvicina a questo frammento un secondo con la lettera A (fig. 12, 4 e lo spessore accumuna anche i frammenti 5 e 6; diversi per spessore e resa della lavorazione i frammenti 2 e 3). Sulla base delle analisi

\footnotetext{
$76 \mathrm{Al}$ momento attuale è l'ipotesi più accreditata. A proposito di misure "oversize» si ricorda anche la testa frammentaria di Dace oggi conservata nei depositi del Teatro di Marcello: se fosse collegata ad una statua, questa svilupperebbe oltre m 4 di altezza! Pergola (scheda cat.) in Parisi Presicce et al., 414, n. 20.

77 Ungaro 2002, 129-133. Di conseguenza, appaiono abbastanza proporzionati alle statue dei Daci: cfr. le perplessità della Ruck 2007, 112, a nostro avviso non condivisibili. Ungaro 2010 b, 105-113.

78 La Rocca 2017, 18-29; Meneghini 2017, $257-$ 262 , e ambedue in questo stesso volume. Cfr. supra note 30 e 71 .

79 Pergola (scheda cat.) in De Nuccio \& Ungaro 2002, 333-335, n. 31: le altre statue analoghe giunte a Firenze col medesimo percorso di quelle della Loggia dei Lanzi sono i due Daci in porfido oggi nel Giar-
}

dino di Boboli. Altri reperti fondamentali sono conservati al Louvre: due statue in porfido rosso e due colonne con ritratti di Traiano e Nerva. Per un esame complessivo resta punto di riferimento essenziale: De Lachenal 1987; De Lachenal 1991. Un corpus lodevole è stato redatto da Francesca Licordari con la tesi di laurea specialistica dal titolo "L'utilizzo del porfido a Roma in età imperiale» (tesi discussa nell'anno accademico 2006/2007, presso Sapienza - Università di Roma- Facoltà di Scienze Umanistiche - Corso di laurea specialistica in Archeologia, relatore prof. Eugenio La Rocca). Fondamentale il catalogo generale sui porfidi messo a punto da Del Bufalo 2012.

80 Inv. nn. FT 6794 e 6824; in Licordari nn. catalogo 34 e 35 .

81 Inv. nn. FT 13201, 13202, 13203, 13204, 13205, 13206; in Licordari 92. 
dei resti pavimentali, sembra possibile che l'aula trisegmentata a sud della piazza possa essere stata pavimentata in porfido, di qui la suggestiva proposta che possa trattarsi dell'edificio noto dalle fonti come la porticus porphiretica ${ }^{82}$; si tratterebbe, come abbiamo accennato, di un settore fondamentale del Foro leggermente più tardo rispetto alla Basilica Ulpia e ai portici della piazza. Acquistano così una luce diversa ai fini dell'attribuzione anche due statue imperiali in porfido, quella togata da Palazzo Nuovo ${ }^{83}$ e quella oggi in Villa Corsini giunta a Firenze col medesimo percorso dei Daci su citati ${ }^{84}$ : possiamo ipotizzare l'altezza ricostruita in almeno $\mathrm{cm} 250 / 260$ in linea con le altre attribuibili a gallerie di sculture ${ }^{85}$.

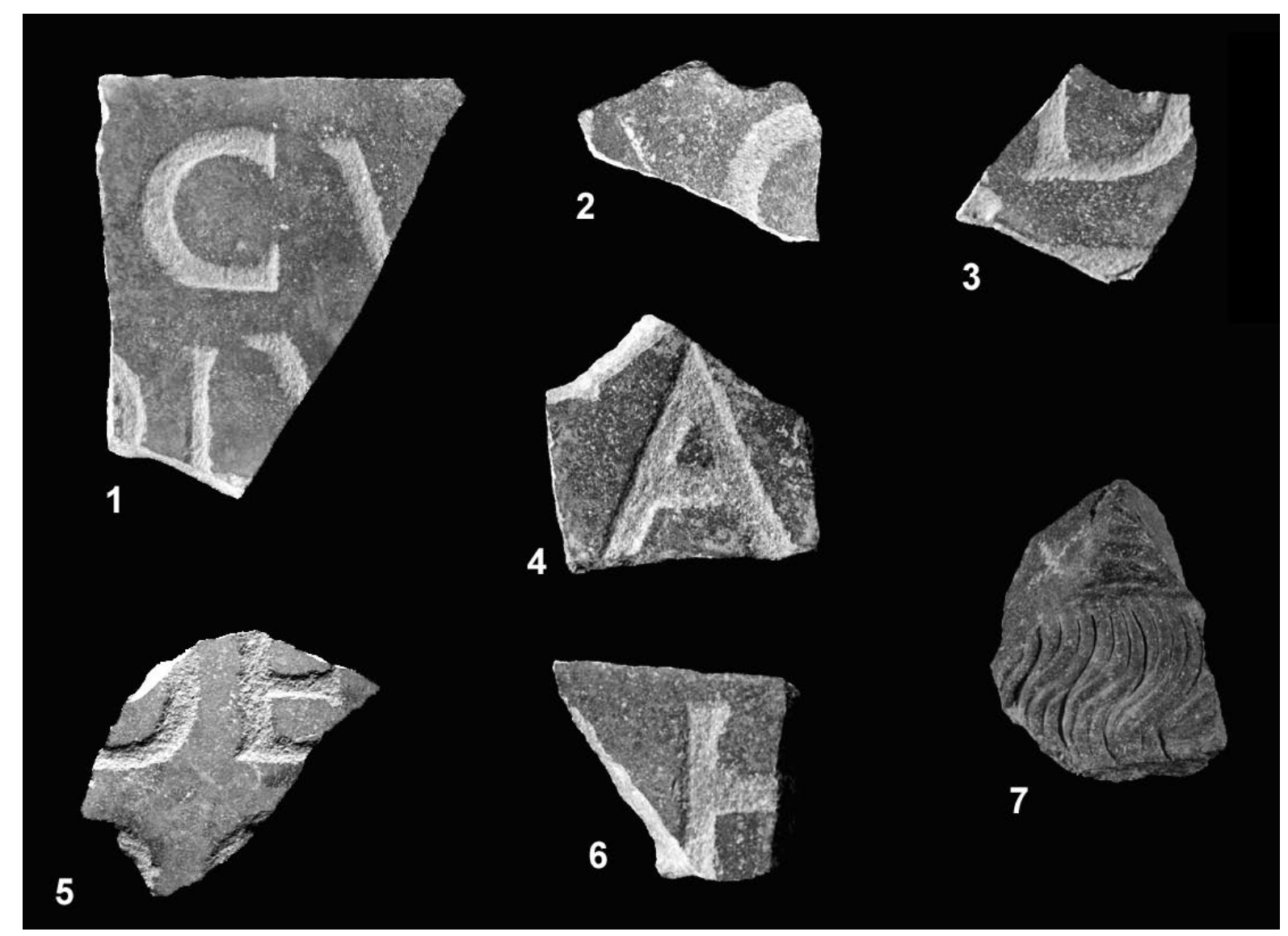

FIG. I 2. Frammenti in porfido, da aula trisegmentata? Frammenti di iscrizioni; frammento di mantello dacico con frangia.

82 Meneghini 2009, 135, fig. 168: le analisi con ripresa macrofotografica hanno permesso allo studioso di avanzare questa affascinante proposta: saremmo di fronte ad una sala di ben $900 \mathrm{mq}$ pavimentata in porfido!

83 Arata 2010, 134-137, n. 7.

${ }^{84}$ Romualdi 2002b (scheda cat.) in De Nuccio \& Ungaro 2002, 330-331, n.29: dalla Collezione Della
Valle-Capranica a quella dei Medici (prima a Roma, poi a Firenze). Vedi supra nota 78.

85 In un deposito dei Mercati di Traiano con materiale sporadico sono conservati piccoli ma preziosi frammenti di sculture non ancora studiati approfonditamente. 
Non è possibile affermare nulla di preciso circa la presenza di statue nella corte sud, cerniera di comunicazione tra il Foro di Traiano e il Foro di Augusto: l'ambiente è rivolto verso il Foro augusteo, la sua decorazione architettonica è sfarzosa, la preziosa iscrizione monumentale ci dice che Traiano è in vita, per realizzarla viene demolita una delle esedre del Foro augusteo ${ }^{86}$. L'importanza del luogo è tale che difficilmente possiamo rassegnarci a leggerlo solo come "corridoio» di transito tra i due complessi!

Del resto Traiano ha avuto nel Foro di Augusto il modello di colossalità, scenografia, comunicazione: il "transito" verso questo modello di riferimento — pur con tutte le diversità- non può essere stato solo un asettico camminamento.

Tutto il programma figurativo del Foro concorre a consolidare e magnificare la personalità dell'imperatore, il quale come abbiamo detto in apertura non ha bisogno di "propaganda», ma "persuade» il suo vasto pubblico di essere il migliore. A questo proposito, significativo un passaggio di Plinio nel rivolgersi a Traiano, all'Optimus Princeps come ad un superiore, curiosamente ammette: «Traiano è un buon imperatore che ci ha ordinato di essere liberi e...noi lo saremo» ${ }^{87}$

\section{BiBLIOGRAFIA}

Albertoni, M., 1993, «Le statue di Giulio Cesare e del Navarca», BCom XCV, 175-183.

Alföldi, G, 1998, "Traianus Pater und die Bauinschrift des Nymphums von Milet», REA 100, n.o 3-4, 367-399.

Amedick, R., 1998, «Ritratto di Traiano su busto loricato», en Bol 1998, 471-472, n. 948.

Arata, P., 2010, «Statua di togato», en E. La Rocca, C. Parisi Presicce, Le sculture di Palazzo Nuovo, 1, Milano: Electa, 134-137.

—, 2017, «Busto di Traiano tipo decennalia», en Parisi Presicce et al. 2017, 387.

Balielo, A., 2017, "Il pane e le cose: la ricchezza e la beneficenza delle donne», en Parisi Presicce et al., 2017, 100-106.

BocCardi, S., (scheda cat.), «Foro di Traiano», en Parisi Presicce et al., 2017, 420, n. 27g.

Bol, P.C., 1998, Forschungen zur Villa Albani: Katalog der antiken Bildwerke V, Berlin: Mann.

Boschung, D. \& W. EcK, «Ein Bildnis der Mutter Traians? Zum Kolossalkopf der sogenanten Agrippina Minor vom Traiansforum», $A A$ 3, 473-481.

Cadario, M., 2004, La corazza di Alessandro: loricati di tipo ellenistico dal IV secolo a.C. al II d.C., Milano: Edizioni LED.

—, 2007, «La decorazione e l'arredo scultoreo nelle basiliche «civili» romane», en F. Sacchi (ed.), La Basilica romana. Genesi e sviluppo tra tarda repubblica e inizio impero, Milano, 95-102.

—, 2011, "L'immagine dell'impero in guerra da Traiano a Marco Aurelio», en La Rocca \& Parisi Presicce 2011, 105-113, scheda cat. I, 1, 260.

Canto, A.M., 2003, Las raices béticas de Trajano: los Trahii de la Itálica turdetana, y otras novedades sobre su familia, Sevilla: Editores Itálica.

Capecchi, G., 1975, «Le statue antiche della Loggia dei Lanzi», BdA 15, 169-178.

Caporossi, S., 2011, «Ritratto di Traiano con egida e corona civica», en La Rocca, Parisi Presicce \& Lo Monaco, 2011, 262, scheda cat. I,4.

Cappellacci, S., M.C. Molinari, 2017, «La monetazione romana sotto il principato di Traiano», en Parisi Presicce et al. 2017, 88-90.

Cenerini, F., 2017, «Le donne di Traiano e la politica», en Parisi Presicce et al. 2017, 107-112.

${ }^{86}$ Meneghini 2009, 137-139; Meneghini 2017,

87 Plin., Panegirico, LIV,5; LXVI,4. 257-262. 
Champlin, E., 1983, «Figlinae Marcianae», Athenaeum 61, 257-264.

Charles-Laforce, M.O., 2015, «Patrimoines et héritages des femmes à Rome: l'exemple des princesses antonines», en C. Courrier, L. Passet (eds.), Arcana Imperii. Mélanges d'histoire économique, sociale et politique, offerts au Professeur Yves Roman, Paris, 233-273.

Chausson, F., 2007, "Variétés généalogiques - IV. Cohésion, collusions, collisions: une autre dynastie antonine», en G. Bonamente, H. Brandt (eds.), Historiae Augustae Colloquium Bambergense, Bari, 123163.

Coarelli, F., 1999, La colonna Traiana, Roma: Ed. Colombo.

Colugnati, G., 2011, "Busto di Traiano tipo decennalia», en: La Rocca, Parisi Presicce \& Lo Monaco 2011, 261, scheda cat. I,3.

Coraggio, F., 2010, «Ritratto di Giulio Cesare», en Gasparri 2010, 62-63, n.37, tav. XXXVI, 1-5.

Del Bufalo, D., Porphyry. Red Imperial Porphyry. Power and Religion, Torino: Allemandi.

De Lachenal, L., 1987, Fortuna dei prigioneri daci a Roma, Xenia Quaderni 8, Roma: De Luca.

—, 1991, "I prigionieri daci della Collezione Medici - Della Valle», en Boboli 90: Atti del Convegno internazionale di studi per la salvaguardia e la valorizzazione del giardino (Firenze, marzo 1989), Firenze: Edifir, 609-621.

De Nuccio, M. \& L. Ungaro, 2002, I marmi colorati della Roma imperiale, catalogo della mostra (Roma 28/09/2002-19/01/2003), Venezia: Marsilio.

Durry, M., 1965, «Sur Trajan père», en Les empereurs romains d'Espagne, Madrid-Italica 1964, Actes du Colloque International organisé par A. Piganiol et H. Terrasse, (CNRS), Paris, 45-53.

Equini Schneider, E., 2017, «Traiano e l'Oriente», en Parisi Presicce et al. 2017, 50-56.

Fittschen, K. \& P. Zanker, 1985, Katalog der römischen Porträts in der Capitolinischen Museen und den anderen kommunalen Sammlungen der Stadt Rom, I. Kaiser und Prinzenbildnisse, Mainz: P. von Zabern.

GaSParri, C., 1979, «Die Gruppe der «Sabinerinnen» in der Loggia dei Lanzi in Florentz», AA 4, 524-543.

—, 2007, «Marmi antichi nella Villa Albani-Torlonia. Appunti per una storia della raccolta», Mouseion. Beiträge zur antiken Plastik, Festschrift für P.C. Bol, Möhnesee, 73-87.

—, 2009-2010, Le sculture Farnese. I-III, Napoli: Electa.

Gonzáles, J., 2017, «Le Guerre Partiche di Traiano: la presa di Ctesifonte», in Parisi Presicce et al., 2017, 65-68.

GonzÁlez Conde-Puente, M.P., 2015, «El proceso de formación de la política dinástica de Trajano», Dialogues d'histoire ancienne, 41, n. ${ }^{\circ} 1,127-148$.

Gross, W.H., 1940, Bildnisse Traians, en Das römische Herrscherbild. II., 2, Berlin: Gebr Mann.

JACKsON, M. et al. 2010, "Composizione e caratteristiche meccaniche dei calcestruzzi della Grande Aula», en L. Ungaro, M.P. del Moro, M. Vitti (eds.), I Mercati di Traiano restituiti. Studi e restauri 2005-2007, Roma: Palombi, 145-154

Kreikeniom, D., 1992, Griechische und römische Kolossalporträts bis zum späten ersten Jahrhundert nach Christus, JdI 27, Berlin: Deutsches Archäologisches Institut.

La Rocca, E., 2017, "Traianus vs Domitianus. Dalla rappresentazione del potere imperiale all'usurpazione dei monumenti pubblici», en Parisi Presicce et al. 2017, 18-29.

La Rocca, E. \& C. Parisi Presicce, 2011, Ritratti: le tante facce del potere, Roma: MondoMostre.

Mansuelli, G., A., 1958-1961, Galleria degli Uffizi: le sculture 1-2, Roma: Istituto Poligrafico dello Stato.

Meneghini, R., 1992, «Roma. Ricerche nel Foro di Traiano. Nuovi dati archeologici e d'archivio riguardanti le vicende medievali del monumento e la chiesa di S. Maria in Campo Carléo", AMediev 19, 409-436.

-, 2009, I Fori Imperiali e i Mercati di Traiano. Storia e descrizione dei monumenti alla luce degli studi e degli scavi recenti, Roma.

—, 2017, «Il Foro di Traiano», en Parisi Presicce et al., 2017, 257-262.

Messa, L. \& L. Ungaro, 1989, «Foro Traiano. Contributi per una ricostruzione storica e architettonica. Rilievi moderni e ricostruzioni, 1926-1986", ArchCl 41, 199-214.

Milella, M., 1995, «Marmi del Foro di Traiano. Elementi architettonici», en L. Ungaro, M. Milella (eds.), 195-241. 
—, 2007, «Il Foro di Traiano», en L. Ungaro (ed.), Il Museo dei Fori Imperiali, Milano: Electa, 192-211.

—, 2017, "Messaggi in pietra nel Foro di Traiano», en Parisi Presicce et al., 2017, 268-274; scheda cat. n.33, 430-431.

Parisi Presicce, C. et al. 2017 (C. Parisi Presicce, M. Milella, S. Pastor \& L. Ungaro), Traiano: costruire l'Impero, creare l'Europa, catalogo della mostra (Roma, 29/11/2017-18/11/2018), Roma: Electa.

Pastor, S., 2017, «Indagini epigrafiche preliminari: la Basilica Ulpia e le legioni di Traiano», en Parisi Presicce et al. 2017, 286-290.

Pergola, S., 2002, «Frammento di statua di dace», en De Nuccio \& Ungaro 2002, 333-335, n. 3.

—, 2017, «Frammento di testa colossale di Dace », en Parisi Presicce et al. 2017, 414, n.20.

Perucchio, R. \& Ph. Brune, 2010, «Il progetto strutturale della Grande Aula e la funzione degli archi di contrasto", en L. Ungaro, M. P. del Moro, M. Vitti (eds.), I Mercati di Traiano restituiti. Studi e restauri 2005-2007, Roma: Palombi, 115-130.

Ratković, D., 2018, «Ritratto di Traianus Pater», en I. Popović, C. Tiussi, M. Verzár (eds.), Tesori e imperatori. Lo splendore della Serbia Romana, Roma, 135.

Rinaldi Tufi, S., 2017, «Traiano in Italia», en Parisi Presicce et al. 2017, 30-38.

Romualdi, A., 2002 a, «Le statue antiche della Loggia dei Lanzi», en Giusti et al., Le statue della Loggia della Signoria a Firenze. Capolavori restaurati, Firenze: Giunti, 17-34.

—, 2002 b, «Statua di imperatore in toga», en De Nuccio \& Ungaro 2002, 330-331, n. 29.

Rose, Ch.B., 1997, Dynastic Commemoration and Imperial Portraiture in the Julio-Claudian Period, Cambridge: Cambridge University Press.

Ruck, B., 2007, Die Grossen dieser Welt: Kolossalporträts im antiken Rom, en Archäologie und Geschichte 11, Heidelberg.

Scheithauer, A., 2000, Kaiserliche Bautätigkeit in Rom: Das Echo in der antiken Literatur, en Habes 32, Stuttgart: Steiner.

Speidel, M.A., 2002, «Bellicosissimus princeps», in A. Nünnerich-Asmus (ed.), Traian: ein Kaiser der Superlative am Beginn einer Umbruchzeit?, Mainz: P. von Zabern, 23-40.

Speranza, E., 2010, «La volta della Grande Aula: analisi critica di ipotesi archeologiche mediante l'uso dei poligoni funicolari», en Ungaro, L., Del Moro, M. P., Vitti, M., (eds.), I Mercati di Traiano restituiti. Studi e restauri 2005-2007, Roma: Palombi, 131-144.

Ungaro, L., 1993, «Foro di Traiano», BCom 95.2, 129-174.

—, 1995, «Marmi del Foro di Traiano. Sculture», en L. Ungaro, M. Milella (eds.), 99-193.

—, 2002, «I Daci dal Foro di Traiano», en M. de Nuccio \& L. Ungaro (eds.) 2002, 129-133.

—, 2005, «Esporre» i Fori Imperiali: ricostruzione, ricomposizione, integrazione, comunicazione nel sistema museale. Le ragioni della conservazione, le ragioni della fruizione», Palladio 36, 69-86.

—, 2008, «Storia, mito, rappresentazione: il programma figurativo del Foro di Augusto e l'Aula del Colosso», en E. La Rocca, P. Leon, C. Parisi Presicce (eds.), Studi di archeologia dedicati a Walter Trillmich, BCom n.s. 18, Roma, 399-417.

—, 2010a, "Introduzione. Il cantiere di restauro tra conservazione e valorizzazione», en L. Ungaro, M.P. del Moro, M. Vitti (eds.), I Mercati di Traiano restituiti. Studi e restauri 2005-2007, Roma: Palombi, 5-12.

—, 2010b, «La Dacia a Roma: statue di Daci e il Foro di Traiano», en E. Oberländer-Târnoveanu, L. Ungaro (eds.), Ori antichi della Romania: prima e dopo Traiano, catalogo mostra, Roma: Silvana Editoriale, 105-113.

—, 2011, "Il cantiere del Foro di Augusto, luogo di sperimentazione e modello formale», in T. Nogales, I. Rodà (eds.), Roma y las provincias: modelo y difusion, Roma, 43-62.

—, 2014a, «La Colonna Traiana nel Foro di Traiano: unità e diversità del messaggio figurativo», en Colonna Traiana-MCM, Atti del Convegno (Roma, 7-8/06/2013), en Ephemeris Dacoromana, serie nuova, XVI, 89-107.

—, 2014b, «La Colonna Traiana e il Foro: la rappresentazione della guerra e della vittoria da Domiziano a Traiano", Los modelos arquitectónicos de Roma y su impacto en las provincias, Actas del XVIII Congreso Internacional de Arqueología clásica (Mérida, 2013), Mérida 2014, 1485-1488. 
—, 2016, «Foro di Augusto, Casa dei Cavalieri di Rodi, Museo dei Fori Imperiali. Ricostruzioni, ricomposizioni, anastilosi dalla Ripartizione X AA.BB.AA. alla Sovrintendenza Capitolina ai Beni Culturali», en L. Abbondanza, L. Ungaro (eds.), La Casa dei Cavalieri di Rodi: Stratigrafia storica di un monumento, BCom 116, 281-310.

—, 2017, schede cat., en Parisi Presicce et al. 2017.

—, 2017a, «Il potere ritratto nel Foro di Traiano», en Parisi Presicce et al. 2017, 91-99.

—, 2017b, «Daci e barbari deportati: dallo stereotipo all'esibizione nel foro, regalità e violenza», en Parisi Presicce et al. 2017, 146-140.

—, 2017c, «Simboli e immagini del trionfo in marmo: «barbari», romani e congeries armorum nel Foro di Traiano", en Parisi Presicce et al. 2017, 291-297.

Ungaro, L. \& M. Milella, 1995 (eds.), I luoghi del consenso imperiale. Il Foro di Augusto. Il Foro di Traiano, Roma: Enel Progetti Museali.

Veyne, P., 2007, L'impero greco-romano. Le radici del mondo globale, Milano 2007.

Virlouvet, C., 1985, «Il Foro di Traiano. Julien Guadet (1834-1908)», en Roma Antiqua. L'area archeologica centrale, Roma: Académie de France à Rome, 184-207.

Vismara, C., 2017, «Traiano e le province africane», en Parisi Presicce et al. 2017, 57-64.

Winkes, R., 1969, Clipeata imago: Studien zu einer römischen Bildnisform, Bonn: R. Habelt.

Wood, S.E., 1999, Imperial Women. A Study in Public Images, 40 BC-AD 68, Leiden: Brill.

Woyteк, B., 2010, Die Reichsprängung des Kaisers Traianus (98-117), en Moneta Imperii Romani 14, Wien: Verlag der Österreichischen Akademie der Wissenschaften.

ZANker, P., 1970, «Das Trajansforum in Rom», JdI 85, 1970, 499-544. 